SU-4240-702

\title{
The Spin-Statistics Connection in Quantum Gravity
}

\author{
A.P. Balachandran ${ }^{1}$, E. Batista ${ }^{2}$, I.P. Costa e Silva ${ }^{3}$ and P. Teotonio-Sobrinho ${ }^{3}$ \\ ${ }^{1}$ Department of Physics, Syracuse University \\ Syracuse, NY 13244-1130, USA \\ ${ }^{2}$ Universidade Federal de Santa Catarina, Centro de Física e Matemática, \\ Dep. MTM, CEP 88.010-970, Florianópolis, SC, Brazil \\ ${ }^{3}$ Universidade de Sao Paulo, Instituto de Fisica-DFMA \\ Caixa Postal 66318, 05315-970, Sao Paulo, SP, Brazil
}

\begin{abstract}
It is well-known that in spite of sharing some properties with conventional particles, topological geons in general violate the spin-statistics theorem. On the other hand, it is generally believed that in quantum gravity theories allowing for topology change, using pair creation and annihilation of geons, one should be able to recover this theorem. In this paper, we take an alternative route, and use an algebraic formalism developed in previous work. We give a description of topological geons where an algebra of "observables" is identified and quantized. Different irreducible representations of this algebra correspond to different kinds of geons, and are labeled by a non-abelian "charge" and "magnetic flux". We then find that the usual spin-statistics theorem is indeed violated, but a new spin-statistics relation arises, when we assume that the fluxes are superselected. This assumption can be proved if all observables are local, as is generally the case in physical theories. Finally, we also discuss how our approach fits into conventional formulations of quantum gravity.
\end{abstract}




\section{Introduction}

The spin-statistics theorem is one of the most fundamental relations in the theories describing the particles of nature. As far as experimental tests are concerned, no elementary particles were ever found which violate it. It was therefore a surprise when it was discovered [1, 2] in the middle 80's that topological geons did violate this relation in general, at least in the case when the spatial topology is not allowed to change. Topological geons are soliton-like excitations of the spatial manifold $\Sigma$ [1, 3]. They can be thought of as lumps of nontrivial topology. For example, in $(2+1) d$, the topology of an orientable, closed surface $\Sigma$ is determined by the number of connected components of $\Sigma$ and by the number of handles on each connected component. Each handle corresponds to a topological geon, i.e., a localized lump of nontrivial topology. It is well known that these solitons have particle-like properties such as spin and statistics. However, as we observed before, unlike ordinary particles they can violate the spin-statistics relation. It has been suggested

[1, 2, 4, 5] that the standard spin-statistics relation can be recovered if one considers processes where geons are (possibly pairwise) created and annihilated, but this necessarily implies a change of the topology of $\Sigma$. In other words, one may have to consider topology change in order to have a spin-statistics theorem for geons [4, 5].

To appreciate the importance of having (or not having) a spin-statistics connection for geons, one must recall that in ordinary quantum field theories in Minkowski space, the particles which arise when we second quantize, for instance, have this connection naturally. Now, in a hypothetic quantum theory of topology, one could think of geons as a kind of "particle", representing excitations of the topology itself. It seems therefore natural to ask about whether they share this connection with "true" particles. As we have mentioned, they do not, but still we find that in the formalism we develop here a different, weaker version of the spin-statistics connection arises.

In the absence of a full-fledged quantum gravity theory, it has become a current practice to consider simple models which retain some of its aspects while being more tractable in the formal aspects. Accordingly, our intention in this work is to use a very simple model, a gauge theory with a finite gauge group in $(2+1) d$ space-time dimensions, to understand the spin-statistics theorem in quantum gravity. This model has the advantage of "isolating" the topological degrees of freedom, which are in a certain sense canonically quantized independently from degrees of freedom coming from metric and other fields. The same model has been considered in a companion paper [6], and there we show that we may consider topology change as a quantum phenomenon depending on the scale of observations. Therefore this model features spatial topology change in some sense. Actually, in spite of the fact that topology change has been inspired by quantum gravity, it has been demonstrated in [7] that it can happen in ordinary quantum mechanics. In this approach, metric is not dynamical, but degrees of freedom related to topology are quantized. The notion of a space with a well defined topology appears only as a classical limit. (See also [8] for related ideas). 
Let us consider a manifold $M$ and some generic field theory (possibly with gauge and Higgs fields) interacting with gravity. It is reasonable to expect that if we could quantize such a complex theory, its observables would give us information on the geometry and topology of $M$. The main point is that one does not need to consider the full theory to get some topological information. It is possible that, in a certain low energy (large distance) limit, there would be a certain set of observables encoding the topological data. We know examples where this is precisely the case. In general, the low energy (large distance) limit of a field theory is not able to probe details of the short distance physics, but it can isolate degrees of freedom related to topology. We may give as an example the low energy limit of $N=2$ Super Yang-Mills, known as the Seiberg-Witten theory [9]. We also have examples of more drastic reduction where a field theory in the vacuum state becomes purely topological [10]. Inspired by these facts we will identify the degrees of freedom, or the algebra $\mathcal{A}^{(n)}$ of "observables", capable of describing $n$ topological geons in $(2+1) d$. Actually, we will argue later in this paper that not all the operators in this algebra are observables in the strict sense. Rather, this algebra is a sort of field algebra (see, for instance, [11] and references therein for more information on field algebras). We say that $\mathcal{A}^{(1)}$ describes a single geon in the same way that the algebra of angular momentum describes a single spinning particle. In this framework what we mean by quantizing the system is nothing but finding irreducible representations of $\mathcal{A}^{(1)}$. As in the case of the algebra of angular momentum, different irreducible representations have to be thought of as different particles. For the moment, we will not be concerned with dynamical aspects. We would like to concentrate on the quantization itself and leave the dynamics to be fixed by the particular model one wants to consider.

An intuitive way of understanding the algebra $\mathcal{A}^{(1)}$ for a topological geon comes from considering a gauge theory with gauge group $G$ in two space dimensions spontaneously broken to a discrete group $H$. For simplicity we will assume that $H$ is finite. As an immediate consequence it follows that the gauge connection (at far distances) is locally flat. In other words, homotopic (based) loops $\gamma$ and $\gamma^{\prime}$ produce the same parallel transport (holonomy). The set of independent holonomies are therefore parametrized by elements $[\gamma]$ in the fundamental group $\pi_{1}(\Sigma)$. It is quite clear that such quantities are enough to detect the presence of a handle. The phase space we are interested in contains only topological degrees of freedom. Therefore such holonomies can be thought of as playing the role of position variables. We also have to take into account the diffeomorphisms (diffeos) that are able to change $[\gamma]$. They will be somewhat the analogues of translations. It is clear that the connected component of the group of diffeomorphisms preserving the basepoint and a frame thereat, the so-called small diffeos, cannot change the homotopy class of $\gamma$. To change the homotopy class of a curve $\gamma$ one needs to act with the so-called large diffeomorphisms. Therefore the analogues of translations have to be parametrized by the large diffeos modulo the small diffeos. This is exactly the mapping class group $M_{\Sigma}$. Also, since we must fix a base point $P$ to define $\pi_{1}(\Sigma)$, we must take into account the fact that the discrete group $H$ can change the holonomies by a conjugation. These three sets of quantities will comprise our algebra $\mathcal{A}^{(1)}$. Contrary to what happens in field 
theory or even in quantum mechanics, we find that $\mathcal{A}^{(1)}$ is finite dimensional. This will be important to avoid technical problems of various kinds. The algebra $\mathcal{A}^{(1)}$ contains the analogue of positions and translations and can be thought as a discrete Weyl algebra. There seems to be no great obstacle to generalize our results also to the case where $H$ is a Lie group [12].

Our algebraic description of geons is analogous to what has been developed for $2 d$ nonabelian vortices by the Amsterdam group [13]. These ideas have been further developed by some of us and coworkers and applied to rings in $(3+1) d$. Their results will not be discussed here since a complete account will be reported in 14.

The algebra encountered by 13 was a special type of Hopf algebra, namely the Drin'feld double of a discrete group [15]. In our case, however, the algebra $\mathcal{A}^{(1)}$ is not Hopf, but it has a Drin'feld double as a subalgebra. For a pair of geons we find that the corresponding algebra $\mathcal{A}^{(2)}$ is closely related to the tensor product $\mathcal{A}^{(1)} \otimes \mathcal{A}^{(1)}$ of single geon algebras. This fact allows us to determine the appropriate algebra $\mathcal{A}^{(n)}$ for an arbitrary number $n$ of geons. Among the elements of $\mathcal{A}^{(2)}$ we find the elements corresponding to the operations of exchanging the positions of two geons and rotating one of them by $2 \pi$. These are the two operations we need in order to answer whether there is a spin-statistics relation. The usual theorem states that the exchange of two identical components (statistics) is equivalent to the rotation by $2 \pi$ of one of its components. It turns out that this is no longer true. However, spin and statistics are not independent but fulfill a well-defined relation.

We would like to point out some differences with respect to the approaches of [2] for the spin-statistics connection. To show their results, the authors of [2] have used anti-particles together with rules for pair creation and annihilation. In our approach the spin-statistics relation follows entirely from the the properties of the field algebras. It is true that we can also have creation and annihilation of geons, but these processes are not directly linked to the spin-statistics relation. For other approaches to the spin-statistics theorem see [16, 17, 18.

One advantage of the algebraic approach is that we can do this analysis without going into the details of the "complete" underlying field theory. We can determine the spectrum $\hat{\mathcal{A}}^{(1)}$ of the geons, i.e., the set of possible irreducible representations of $\mathcal{A}^{(1)}$, but a particular field theory may restrict the available possibilities in $\hat{\mathcal{A}}^{(1)}$. The determination of these possibilities requires the study of particular examples of the underlying field theories. That may be a very difficult task. In this paper our intention is to use the simplified algebraic "field" theory and see what it can teach us. It is remarkable that such a simple framework can reveal important features of quantum geons such as a constraint involving spin and statistics. Rules for quantum topology change are discussed in a companion paper [6].

There is a systematic way to incorporate our algebraic methods in conventional approaches to quantum gravity. When that is done, we end up selecting a particular class of vector bundles, the sections of which are state vectors of quantum gravity (they specify 
domains of operators like the Hamiltonian). We shall discuss these issues in detail elsewhere, limiting ourselves to a concise discussion in Section 6 in this paper. The present paper therefore can be interpreted as sponsoring the use of these bundles in quantizing gravity. We think that there are powerful reasons supporting this point of view. Indeed our work here shows that these bundles nicely incorporate information on classical spatial topology and imply a (generalized) spin-statistics theorem, whereas if this selection of bundles is abandoned, there are many possible choices of bundles in the presence of geons, and most do not imply any sort of spin-statistics connection.

This paper is organized as follows. The field algebras $\mathcal{A}^{(n)}$ are described in Section 2. In particular, the representations of $\mathcal{A}^{(1)}$ will play an important role when we discuss the spin-statistics connection. Quantization of the system is given in Section 3. In this section we are able to classify the irreducible representations for a class of algebras $\tilde{\mathcal{A}}$ that includes our algebra of interest as a particular example. It is important to note that these Sections are shortened copies of Sections in the companion paper [6], which we reproduce here for the benefit of the reader, rendering this paper basically self-contained. The original part is in the following Sections. The existence of a novel spin-statistics connection for $2 d$ orientable geons is established in Section 4 , under certain asumptions which become clear in Section 5, with the introduction of the property of clustering for a system of $N$ geons, and superselection of the global fluxes of geons. Section 6 explores how one can use the representations of the algebra of observables for geons to obtain geon states in quantum gravity. The paper ends with some general remarks and an outlook on future work.

\section{The Algebras for $(2+1) d$ Topological Geons}

Throughout this work our setting is a space-time of the form $\Sigma \times \mathbb{R}$. Here, the spatial manifold $\Sigma$ is two-dimensional, and will be typically assumed to be a plane with one or several handles. Topological geons in this $(2+1) d$ context are simply (for orientable space-times) these handles on the spatial manifold (for a more detailed account and a more general definition of geons see, for instance, [1, 6, 19]). Our aim in this section is to define some "observables" which describe the topological character of a geon. As we will see later, the algebras we obtain contain some operators which are not really observables, since they represent non-local operators which are in a certain sense "gauged away" whenever we perform physical measurements for geons. Thus we will refer to the kind of algebra we will encounter as a field algebra [11].

The presentation of the field algebra of geons given here will not be detailed. The reader is refered to [6] for a more comprehensive discussion.

We will follow an approach inspired by the work of the Amsterdam group, which is reported in ref. [13]. In this work, the group investigates the properties of vortex solutions 
of a $(2+1) d$ gauge field theory in Minkowski spacetime where the gauge symmetry of a Lie group $G$ is spontaneously broken to a finite group $H$ by a non-vanishing expectation value of a Higgs field $\Phi$. See [13] for details. The Lagrangian is given by

$$
\mathcal{L}=\frac{1}{4} F_{\mu \nu}^{a} F_{a}^{\mu \nu}+\operatorname{Tr}\left[\left(D_{\mu} \Phi\right)^{*} \cdot\left(D^{\mu} \Phi\right)\right]-V(\Phi)
$$

where $\mu, \nu=0,1,2$, and $a$ is a Lie algebra index. For simplicity, we assume that $G$ is connected and simply connected. The fields $F_{\mu \nu}^{a}$ are the components of the field strength of the Yang-Mills potential $A_{\mu}^{a}$ and $D_{\mu}$ denotes the covariant derivative determined by this potential. The Higgs field $\Phi$ is in the adjoint representation and can be expanded in terms of generators $T^{a}$ of the Lie algebra of $G$, and $V(\Phi)$ is a $G$-invariant potential. In this paper we shall be concerned with the low energy, or equivalently, the long range behavior of this theory, in the temporal gauge $A_{0}^{a}=0$. This is obtained by minimizing the three terms in the energy density separately. Minimizing the term corresponding to the energy density of the Yang-Mills field, we obtain the condition $F_{\mu \nu}^{a}=0$, from which we conclude that we are dealing only with flat connections. The minimum of the potential restricts the values of the Higgs field to the vacuum manifold, which is invariant by $H$. Finally, the condition $D \Phi=0$, required for minimizing the energy density from the second term, tells us that the holonomies

$$
\tau(\gamma)=P \exp \left\{\int_{\gamma} A_{i}^{a} T_{a} d s^{i}\right\} ; i \in\{1,2\}
$$

take values in the finite group $H$.

Here and in what follows we will fix a base point $P$ for loops, so that all loops will begin and end at $P$.

This gauge theory may have topologically non-trivial, static solutions such as vortices. It is very well known that the core radii of these vortices are inversely proportional to the mass of the Higgs boson, and therefore they may be viewed as point-like in the low-energy regime of the theory. Hence, according to a standard argument, to describe the $N$-vortex solutions we may consider solutions for the vortex equations

$$
\begin{aligned}
F_{i j}^{a} & =0 \\
D_{i} \Phi & =0 \\
V(\Phi) & =0
\end{aligned}
$$

on a spacetime of the form $\Sigma \times \mathbb{R}$, where $\Sigma$ is the plane with $N$ punctures, playing the role of the vortices.

One way to explain our approach is based on a field theory like (2.1). Addition of gravitational terms to (2.1) would not affect our arguments. The difference in our approach is that we shall work in the zero vortex number sector of this theory, but on a plane with geons. Hence, instead of puncture, the non-trivial topology is characterized by handles. Now, take a solution $(A, \Phi)$ for the vortex equations (2.3). By fixing a 
point $P \in \Sigma$, the holonomy of $A$ around any path $\gamma$ based at $P$ depends only on its homotopy class, since $A$ is flat. It takes values in a subgroup $H$ of $G$, which preserves the vacuum manifold, in view of the equations for $\Phi$ [13]. Therefore, any solution of the vortex equations determines a homomorphism $\tau$,

$$
\tau: \pi_{1}(\Sigma) \rightarrow H
$$

of the fundamental group $\pi_{1}(\Sigma)$ to the group $H$. Conversely, given such a homomorphism $\tau$ we can define a solution for eqs.(2.3) in the following way. Take the universal covering space $\tilde{\Sigma}$ of $\Sigma$. It is the total space of a principal bundle over $\Sigma$ with structure group $\pi_{1}(\Sigma)$. Via the homomorphism $\tau$ we can construct an associated principal $H$-bundle over $\Sigma$, which is a subbundle of the original $G$-bundle. Since $H$ is finite, this bundle has a unique flat connection $A_{i}^{a}$, which can be viewed as a reducible connection on the $G$-bundle. We now find a $\Phi$. After fixing some $\Phi_{0}$ in the vacuum manifold, we define $\Phi(P)=\Phi_{0}$. Then, since $\Phi$ must be covariantly constant, its value can be obtained for each $x \in \Sigma$ by parallel transporting $\Phi_{0}$ along some path from $P$ to $x$ in $\Sigma$ :

$$
\Phi(x)=P \exp \left\{\int_{P}^{x} A_{i}^{a} T_{a} d s^{i}\right\} \Phi_{0} .
$$

The pair $\left(A_{i}^{a}, \Phi\right)$ thus constructed is obviously a solution of the vortex equations. Therefore the space of solutions for the vortex eqs. (2.3) is essentially parametrized by homomorphisms $\tau: \pi_{1}(\Sigma) \rightarrow H$. Each such homomorphism is then a vortex configuration when we have punctures. In our context, we will call one such homomorphism a geon configuration. In general, it gives non-zero "magnetic fluxes" around non-trivial elements of $\pi_{1}(\Sigma)$.

The finite group $H$ acts on the space of solutions. In terms of homomorphisms we have that, under these $H$-transformations, a flux $\sigma$ transforms as

$$
\sigma \mapsto h \sigma h^{-1}
$$

In other words, we have an action of $H$ by conjugation of the fluxes. We shall simply refer to this action as the $H$-transformations. The group elements $h \in H$ will be regarded as operators when we quantize the theory, also denoted by $h$. The multiplication of two $H$-transformations is the same as the group multiplication. Therefore the algebra of such operators turns out to be the group algebra $\mathbb{C}(H)$.

As for the physical interpretation of the $H$-tranformations we note that the mathematical action depicted in (2.6) is entirely equivalent, from a physical standpoint, to what occurs when one makes a flux $\sigma$ encircle a source of flux $h$ at infinity. Since such an operation is non-local, one must conclude that the $H$-transformations cannot be considered local in the theory, i.e., cannot be implemented by local operators.

The total algebra in the case of vortices (punctures)is the semi-direct product $D(H)=$ $\mathbb{C}(H) \ltimes \mathcal{F}(H)$, where $\mathcal{F}(H)$ is the algebra of complex-valued functions on $H$ with product given by pointwise multiplication: it describes the "position observables" for a vortex. 
The reason for this denomination will become clear when we discuss geons. The algebra $D(H)$ is the so-called Drin'feld double [13]. It has the structure of a quasi-triangular Hopf algebra. The Hopf structure [15] means in particular the existence of a co-product, i.e, a map

$$
\Delta: D(H) \longrightarrow D(H) \otimes D(H),
$$

which is a homomorphism of algebras. In [13] the fluxes are seen as particles in $(2+1) d$ and are then first quantized: the (internal) Hilbert space $\mathcal{H}$ is constructed, and the elements of the algebra $D(H)$ act as operators on this Hilbert space. $\mathcal{H}$ decomposes into irreducible representations of $D(H)$, corresponding to the different particle sectors of the quantum theory. The existence of a co-product allows one to understand fusing processes between particles. The quasi-triangularity implies the existence of the $R$-matrix, $R \in D(H) \otimes D(H)$, responsible for all braiding processes between particles. Again, for further details see [13].

How is the topology of $\Sigma$ taken into account in this approach? First of all, we have seen that the physically distinct (for vortices and/or geons) configurations are in one-to-one correspondence with conjugacy classes of homomorphisms of $\pi_{1}(\Sigma)$ into $H$. Moreover, it is well known that for a finite group $H$ the elements in the latter space are in oneto-one correspondence with equivalence classes of principal $H$-bundles over $\Sigma$. Therefore the only degree of freedom in this theory is the topology of these bundles. Second, a configuration for which the holonomy is trivial around some puncture or handle is indistinguishable, from the standpoint of the low-energy theory, to another in which that particular puncture or handle is absent. Therefore the low-energy theory somehow actually allows for "topology fluctuations" of $\Sigma$ as long as we stay within its limits. Such a way of viewing topology change is explored in [6]. It is very much akin to the views pursued in non-commutative geometry, where one uses an algebra to encode space-time geometry and topology. In this approach the usual "classical" view of a background manifold is secondary, and the topology is actually viewed as a consequence of the algebraic setting one uses.

In order to determine the field algebra for a topological geon, we will first try to find the analogues of the "position observables" for a geon. Now, $\Sigma$ is the plane with one or more handles, and for simplicity we shall assume throughout that there are no vortices, i.e., we work in the zero vortex number sector of the low-energy limit of the theory given by the Lagrangian in (2.1). In this case, all non-trivial configurations will be related solely to holonomies around and through the handles.

Let us start by taking $\Sigma$ to be the plane with a handle. On all figures, a geon will be thought of as a square hole on the plane, with the opposite sides identified. One can show that $\pi_{1}(\Sigma)$ has two generators $\left[\gamma_{1}\right]$ and $\left[\gamma_{2}\right]$, shown by Fig. 2.1. It can be shown that

$$
\left[\gamma_{3}\right]=\left[\gamma_{1}\right]\left[\gamma_{2}\right]\left[\gamma_{1}\right]^{-1}\left[\gamma_{2}\right]^{-1}
$$

Actually, $\pi_{1}(\Sigma)$ is freely generated by $\left[\gamma_{1}\right]$ and $\left[\gamma_{2}\right]$. Let $g=W\left(\left[\gamma_{1}\right],\left[\gamma_{2}\right]\right) \in \pi_{1}(\Sigma)$, be a word in $\left[\gamma_{1}\right],\left[\gamma_{2}\right]$ and their inverses. Then $\tau$ maps $g$ to $W(a, b) \in H$ where $a=\tau\left(\gamma_{1}\right)$ and 


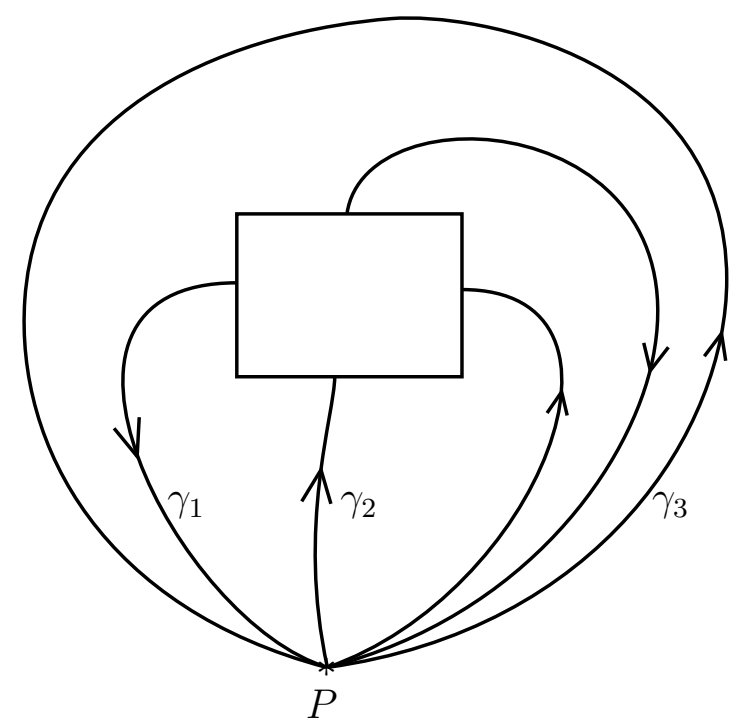

Fig. 2.1: The figure shows the loops $\gamma_{i}(1 \leq i \leq 3)$. The homotopy classes $\left[\gamma_{1}\right]$ and $\left[\gamma_{2}\right]$ generate the fundamental group. The class $\left[\gamma_{3}\right]$ is not independent of $\left[\gamma_{1}\right]$ and $\left[\gamma_{2}\right]$.

$b=\tau\left(\gamma_{2}\right)$. Therefore the map $\tau: \pi_{1}(\Sigma) \rightarrow H$ is completely characterized by the fluxes $\tau\left(\gamma_{1}\right)=a$ and $\tau\left(\gamma_{2}\right)=b$. Since there is no relation between $a$ and $b$, the set $T$ of all maps is labeled by $H \times H$.

We now define more precisely what we mean by a geon configuration. Let $H$ be a finite group and $\Sigma$ the plane with one geon, i.e., a two dimensional manifold given by

$$
M=\mathbf{R}^{2} \# \mathbf{T}^{2} .
$$

Let $\gamma_{1}$ and $\gamma_{2}$ denote representative loops whose classes generate $\pi_{1}(\Sigma)$. We define a classical configuration $\tau_{(a, b)} \in T$ of a geon as the homomorphism defined by

$$
\tau_{(a, b)}\left(\gamma_{1}\right)=a, \text { and } \tau_{(a, b)}\left(\gamma_{2}\right)=b
$$

It is important to bear in mind that $T \cong H \times H$ and therefore that it is a finite discrete set. For simplicity of notation, a geon configuration will be denoted simply by a pair $(a, b)$ of fluxes. Note that we are not explicitly identifing those configurations which differ by an $H$-transformation. This is because wave functions need only be "covariant" under the symmetries of the problem, and only its modulus squared and other observable quantities, like Aharonov-Bohm phases, must be invariant. In our approach, this will happen naturally, just as in 13 .

With $T \cong H \times H$ being the configuration space for a geon, the corresponding algebra of "position observables" is $\mathcal{F}(T)$, the algebra of complex-valued functions on $T$ with product given by pointwise multiplication. Instead of working with the abstract algebra, we specify a representation. Let $V$ be the (finite-dimensional) complex vector space 
generated by the vectors $|a, b\rangle, a, b \in H$. We will call the representation on $V$, to be defined below, the defining representation. The algebra $\mathcal{F}(T)$ is generated by projectors on $V$ denoted by $Q_{(a, b)}$. They are defined by

$$
Q_{(a, b)}|c, d\rangle=\delta_{a, c} \delta_{b, d}|c, d\rangle \text {. }
$$

The operator $Q_{(a, b)}$ represents a "delta function" supported at $(a, b)$, i.e., it gives 1 when evaluated on $(a, b)$, and zero everywhere else. Indeed, from (2.8) one finds that

$$
Q_{(a, b)} Q_{(c, d)}=\delta_{a, c} \delta_{b, d} Q_{(c, d)}
$$

Besides the projectors $Q_{(a, b)}$, which play the role of position operators in ordinary quantum mechanics, we have also some operators capable of changing $(a, b)$. They are somewhat analogous to momentum operators. For example, like in the case of vortices, $H$-transformations act on the configurations. It turns out that for a geon there are additional operators besides $H$-transformations. They correspond to the action of the group Dif $f^{\infty}(\Sigma)$ of diffeomorphisms of $\Sigma$ that keeps infinity invariant.

We will start by first examining the $H$-transformations.

The group $H$ acts on $T$ simply by conjugating both fluxes in $(a, b)$. This will induce an operator $\hat{\delta}_{g}$ for each $g \in H$, acting on the defining representation $V$ by

$$
\hat{\delta}_{g}|a, b\rangle=\left|g a g^{-1}, g b g^{-1}\right\rangle .
$$

From (2.10) one sees that the multiplication of operators $\hat{\delta}_{g}$ is given by

$$
\hat{\delta}_{g} \hat{\delta}_{h}=\hat{\delta}_{g h}
$$

The corresponding algebra generated by $\delta_{g}$ is the group algebra $\mathbb{C}(H)$. The relation between $\mathcal{F}(H \times H)$ and $\mathbb{C}(H)$ can be derived from (2.8) and (2.10). One sees immediately that

$$
\hat{\delta}_{g} Q_{(a, b)} \hat{\delta}_{g}^{-1}=Q_{\left(g a g^{-1}, g b g^{-1}\right)} .
$$

In other words, the algebra $\mathbb{C}(H)$ acts on $\mathcal{F}(H \times H)$.

Besides $H$-transformations, fluxes $(a, b)$ can change under the action of the group Dif $f^{\infty}(\Sigma)$. It is clear that elements belonging to the subgroup Dif $f_{0}^{\infty}(\Sigma)$, the component connected to identity, act trivially on $\pi_{1}(\Sigma)$ 円and hence on $(a, b)$. Therefore what matters is the action of the so-called mapping class group $M_{\Sigma}$ [20, 21], defined as

$$
M_{\Sigma}=\frac{\operatorname{Diff} f^{\infty}(\Sigma)}{\operatorname{Diff_{0}^{\infty }(\Sigma )} .}
$$

For the present case, $\Sigma$ is the plane with a single geon and the mapping class group is isomorphic to the central extension of the group $S L(2, \mathbf{Z})$, denoted by $S t(2, \mathbf{Z})$ and

\footnotetext{
${ }^{1}$ For simplicity, we take the basepoint $P$ to be at infinity.
} 


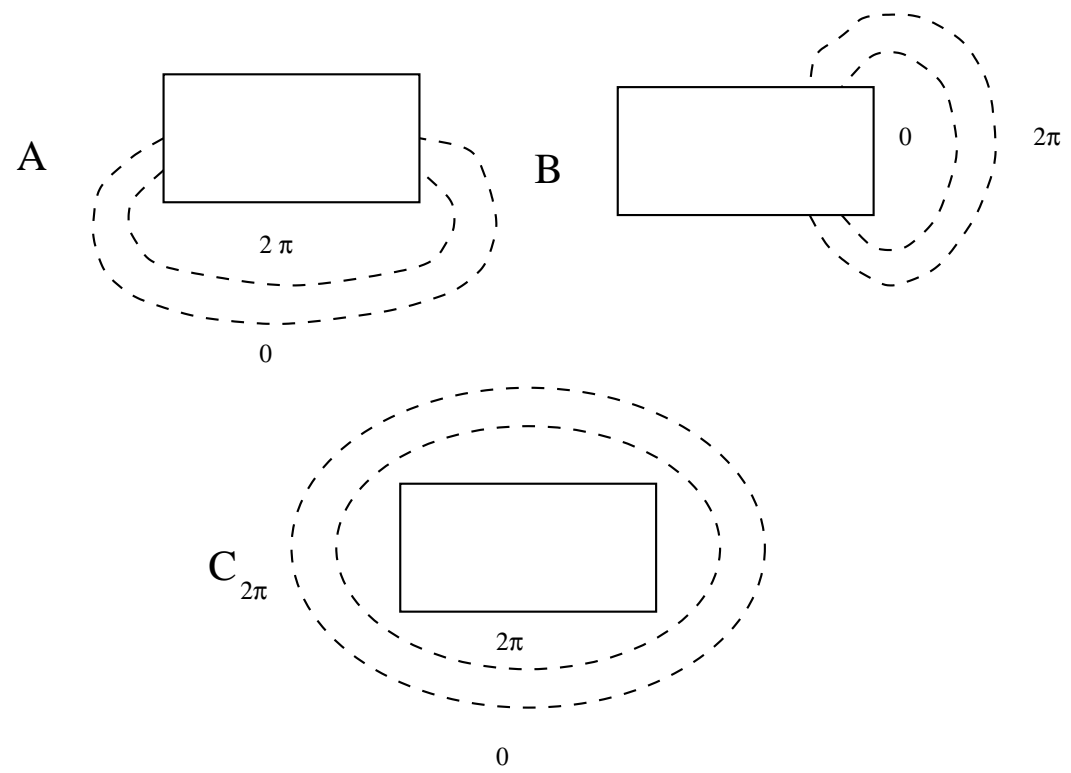

Fig. 2.2: Dehn twists corresponding to diffeomorphisms of the mapping class group. The annuli enclose loops, which we have omitted in the figure. Rotations are counterclockwise by convention.

called the Steinberg group. This is the same as the mapping class group of a torus minus one point [19]. We denote generators of $M_{\Sigma}=S t(2, \mathbf{Z})$ by $A$ and $B$. They correspond to (isotopy classes of) diffeomorphisms [ called Dehn twists. A Dehn twist is realized as follows. Take a loop in $\Sigma$. Then draw an annulus enclosing the loop and introduce radial coordinates $r \in[0,1]$, with $r=0$ and $r=1$ corresponding to the boundaries of the annulus, see Fig. 2.2. Then rotate the points of the annulus in such a way that the angle of rotation $\theta(r)$ is zero for $r=0$ and gradually increases, becoming $2 \pi$ at $r=1$. Figure 2.2 shows how to produce Dehn twists, and in Fig. 2.3, we show how the Dehn twist $B$ deforms the loop $\gamma_{1}$. There is also the Dehn twist along a loop enclosing the geon, which can be interpreted as the $2 \pi$-rotation of the geon [1, 3, 19]. It will be important when we discuss spin of the geon. The corresponding diffeo is denoted by $C_{2 \pi}$ in Fig. 2.2. However, $C_{2 \pi}$ is not independent of $A$ and $B$. One can show that 19]

$$
C_{2 \pi}=\left(A B^{-1} A\right)^{4} \text {. }
$$

The group $M_{\Sigma}$ is generated by $A$ and $B$, with the relation that $C_{2 \pi}$ commutes with $A$ and $B$. It is useful to think of the elements of $M_{\Sigma}$ as words $W(A, B)$ in $A, B$ and their inverses.

The action of $A$ and $B$ on $\left[\gamma_{i}\right] \in \pi_{1}(\Sigma)$ induces an action on $(a, b) \in T$, and therefore induces operators $\hat{A}$ and $\hat{B}$ in the defining representation acting on $V$. Let us take as an example the action of $B$ on $\gamma_{1}$, as given by Fig. 2.3. One sees that $\left[\gamma_{1}\right] \rightarrow\left[\gamma_{1}\right]\left[\gamma_{2}\right]$, and therefore $a \rightarrow a b$. On the other hand, $B$ keeps $\left[\gamma_{2}\right]$ invariant. One can verify that $A$ and

\footnotetext{
${ }^{2}$ One can see from (2.13) that the mapping class group consists of isotopy classes of diffeomorphisms. Throughout this paper we shall loosely use a representative in a class as the class itself.
} 


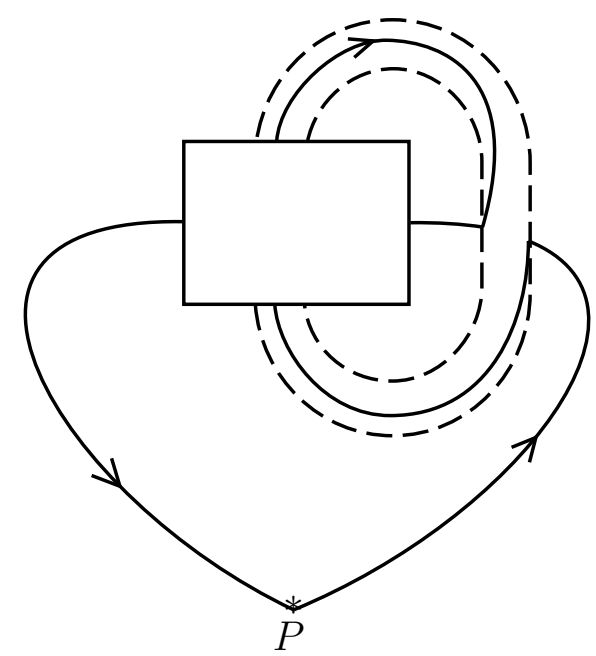

Fig. 2.3: Dehn twist $B$ and its action on $\gamma_{1}$.

$B$ induce the following operators:

$$
\begin{aligned}
& \hat{A}|a, b\rangle=|a, b a\rangle, \\
& \hat{B}|a, b\rangle=|a b, b\rangle,
\end{aligned}
$$

For an arbitrary word $W(A, B)$, the corresponding operator is $W(\hat{A}, \hat{B})$, i.e., the same word but with $A$ and $B$ replaced by $\hat{A}$ and $\hat{B}$. For example, the Dehn twist $C_{2 \pi}$ of Fig. 2.2 is written as $\left(A B^{-1} A\right)^{4}$ and the corresponding operator $\hat{C}_{2 \pi}$ can be immediately computed to be

$$
\hat{C}_{2 \pi}|a, b\rangle=\left|\mathbf{c}^{-1} a \mathbf{c}, \mathbf{c}^{-1} b \mathbf{c}\right\rangle
$$

where $\mathbf{c}=a b a^{-1} b^{-1}$.

The algebra generated by the operators $\hat{A}$ and $\hat{B}$ is the group algebra $\mathbb{C}(\mathcal{M})$. Together with $\mathbb{C}(H)$ and $\mathcal{F}(H \times H)$ it gives us the total algebra $\mathcal{A}^{(1)}$ for a single topological geon. From the definitions (2.8), (2.10) and (2.15) one sees that

$$
\begin{gathered}
\hat{\delta}_{g} \hat{A}=\hat{A} \hat{\delta}_{g}, \quad \hat{\delta}_{g} \hat{B}=\hat{B} \hat{\delta}_{g}, \\
\hat{\delta}_{g} Q_{(a, b)} \hat{\delta}_{g}^{-1}=Q_{\left(g a g^{-1}, g b g^{-1}\right)} \\
\hat{C}_{2 \pi} \hat{A}=\hat{A} \hat{C}_{2 \pi}, \quad \hat{C}_{2 \pi} \hat{B}=\hat{B} \hat{C}_{2 \pi}, \\
\hat{A} Q_{(a, b)} \hat{A}^{-1}=Q_{(a, b a)}, \quad \hat{B} Q_{(a, b)} \hat{B}^{-1}=Q_{(a b, b)} .
\end{gathered}
$$

Therefore, both algebras $\mathbb{C}(H)$ and $\mathbb{C}(\mathcal{M})$ act on $\mathcal{F}(H \times H)$. The action of a generic word $W(\hat{A}, \hat{B})$ on $Q_{(a, b)}$ will be denoted by

$$
W(\hat{A}, \hat{B}) Q_{(a, b)}=Q_{\left(w^{(a)}, w^{(b)}\right)} W(\hat{A}, \hat{B}) .
$$


where $\left(w^{(a)}, w^{(b)}\right)$ is a pair of words in $a$ and $b$ and their inverses, representing the action of $W(A, B)$ on $(a, b)$.

There are two equivalent ways of presenting $\mathcal{A}^{(1)}$. One is by using the defining representation of (2.8), (2.10) and (2.15). Another way is to define $\mathcal{A}^{(1)}$ as the algebra generated by $Q_{(a, b)}, \hat{\delta}_{g}, \hat{A}$ and $\hat{B}$ with the relations (2.17). In any case, we have that

$$
\mathcal{A}^{(1)}=\mathbb{C}(H \times \mathcal{M}) \ltimes \mathcal{F}(H \times H) .
$$

We may introduce the algebra for two topological geons following exactly the same ideas as for a single topological geon. We will briefly outline here the main constructions. For details, see [6]. We recall that for a single geon, $\mathcal{A}^{(1)}$ consists of three sub-algebras, generated by the "position observables" $\mathcal{F}(T)$, the $H$-transformations $\mathbb{C}(H)$, and the "translations", i.e., a realization $\mathcal{M}$ of the mapping class group $M_{\Sigma}$. The algebra $\mathcal{A}^{(2)}$ for two geons will consist of the same three distinct parts, with $T=H \times H \times H \times H \equiv H^{4}$ and $\Sigma$ replaced by a plane with two handles.

It is natural to work with the defining representation on $V \otimes V$ spanned by vectors of the form

$$
\left|a_{1}, b_{1}\right\rangle \otimes\left|a_{2}, b_{2}\right\rangle
$$

where the subscripts denote the respective geons. The "position observables" are generated by projectors $Q_{\left(a_{1}, b_{1}\right)} \otimes Q_{\left(a_{2}, b_{2}\right)}$ acting on $V \otimes V$ in the obvious way, i.e.,

$$
Q_{\left(a_{1}, b_{1}\right)} \otimes Q_{\left(a_{2}, b_{2}\right)}\left|a_{1}^{\prime}, b_{1}^{\prime}\right\rangle \otimes\left|a_{2}^{\prime}, b_{2}^{\prime}\right\rangle=\delta_{a_{1}, a_{1}^{\prime}} \delta_{b_{1}, b_{1}^{\prime}} \delta_{a_{2}, a_{2}^{\prime}} \delta_{b_{2}, b_{2}^{\prime}}\left|a_{1}, b_{1}\right\rangle \otimes\left|a_{2}, b_{2}\right\rangle .
$$

Therefore, the "position" operators belong to $\mathcal{A}^{(1)} \otimes \mathcal{A}^{(1)}$.

The action of an $H$-transformation $g \in H$ on the fluxes $\left(a_{1}, b_{1}, a_{2}, b_{2}\right)$ is by a global conjugation. This induces the action

$$
\left|a_{1}, b_{1}\right\rangle \otimes\left|a_{2}, b_{2}\right\rangle \rightarrow\left|g a_{1} g^{-1}, g b_{1} g^{-1}\right\rangle \otimes\left|g a_{2} g^{-1}, g b_{2} g^{-1}\right\rangle
$$

on $V \otimes V$. The corresponding operator is obviously identified with $\hat{\delta}_{g} \otimes \hat{\delta}_{g} \in \mathbb{C}(H) \otimes \mathbb{C}(H)$, since

$$
\hat{\delta}_{g} \otimes \hat{\delta}_{g}\left|a_{1}, b_{1}\right\rangle \otimes\left|a_{2}, b_{2}\right\rangle=\left|g a_{1} g^{-1}, g b_{1} g^{-1}\right\rangle \otimes\left|g a_{2} g^{-1}, g b_{2} g^{-1}\right\rangle
$$

Hence, $H$-transformation operators also belong to $\mathcal{A}^{(1)} \otimes \mathcal{A}^{(1)}$.

We now start to consider the action of the mapping class group $M_{\Sigma}$. For two or more geons, $M_{\Sigma}$ is much more complicated than for a single geon 20]. The mapping class group is generated by Dehn twists of the type $A$ and $B$ (see Fig. 2.2) for each individual geon together with diffeomorphisms involving pairs of geons.

Let $A_{i}, B_{i}, i=1,2$ be the generators of the "internal diffeos" for each individual geon. The corresponding operators acting on $V \otimes V$ are clearly given by

$$
\hat{A}_{1}=\hat{A} \otimes \mathbb{I}, \quad \hat{A}_{2}=\mathbb{I} \otimes \hat{A}
$$




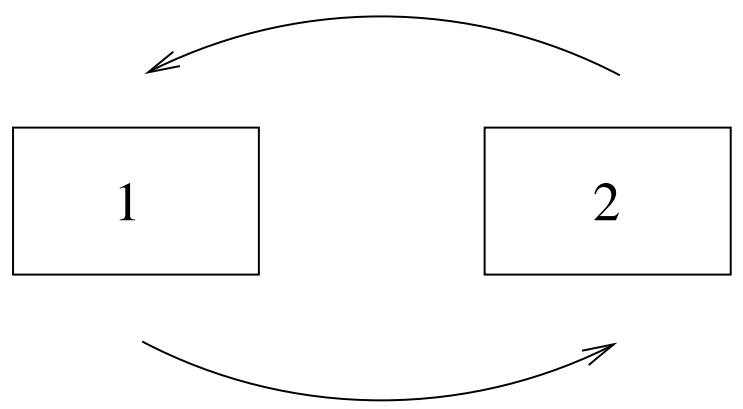

Fig. 2.4 Geon exchange.

$$
\hat{B}_{1}=\hat{B} \otimes \mathbb{I I}, \quad \hat{B}_{2}=\mathbb{I} \otimes \hat{B}
$$

where II is the identity operator on $V$.

There are two additional classes of transformations besides the internal diffeos. The first one, called exchange, is the analogue of the elementary braiding of two particles. The second, called handle slide, has no analogue for particles, since it makes use of the internal structure of the geon.

So far, all operators in the algebra for $\mathcal{A}^{(2)}$ were of the form $x \otimes y \in \mathcal{A}^{(1)} \otimes \mathcal{A}^{(1)}$. It turns out that this is not the case for exchanges and handle slides. They correspond somewhat to interactions and cannot be written strictly in terms of operators in $\mathcal{A}^{(1)} \otimes \mathcal{A}^{(1)}$. In order to describe interactions between geons, we need to define a pair of flip automorphisms of $V \otimes V$. They are necessary in the construction of the exchange and handle slide operators.

Definition: Given a two geon state

$$
\left|a_{1}, b_{1}\right\rangle \otimes\left|a_{2}, b_{2}\right\rangle \in V \otimes V,
$$

the flip automorphisms $\sigma$ and $\gamma$ are defined by:

$$
\begin{aligned}
\sigma\left|a_{1}, b_{1}\right\rangle \otimes\left|a_{2}, b_{2}\right\rangle & :=\left|a_{2}, b_{2}\right\rangle \otimes\left|a_{1}, b_{1}\right\rangle, \\
\gamma\left|a_{1}, b_{1}\right\rangle \otimes\left|a_{2}, b_{2}\right\rangle & :=\left|a_{1}, b_{2}\right\rangle \otimes\left|a_{2}, b_{1}\right\rangle .
\end{aligned}
$$

Both are not given geometrically as morphisms of the mapping class group, but unless one introduces these operators, the algebra of two geons cannot be related directly to the algebras for a single geon. We will show that the algebra $\mathcal{A}^{(2)}$ can be obtained from the tensor product $\mathcal{A}^{(1)} \otimes \mathcal{A}^{(1)}$ when we add $\sigma$ and $\gamma$.

In the exchange process, two geons permute their positions. In our convention, the geon on the right (left) moves counterclockwise to the position of the left(right) (see Fig. 2.4). The effect of a geon exchange on the states is of the form

$$
\mathcal{R}\left|a_{1}, b_{1}\right\rangle \otimes\left|a_{2}, b_{2}\right\rangle=\left|\mathbf{c}_{1}^{-1} a_{2} \mathbf{c}_{1}, \mathbf{c}_{1}^{-1} b_{2} \mathbf{c}_{1}\right\rangle \otimes\left|a_{1}, b_{1}\right\rangle,
$$




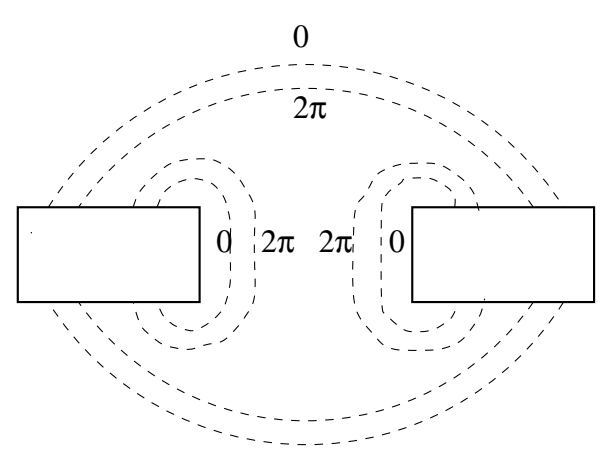

(a)

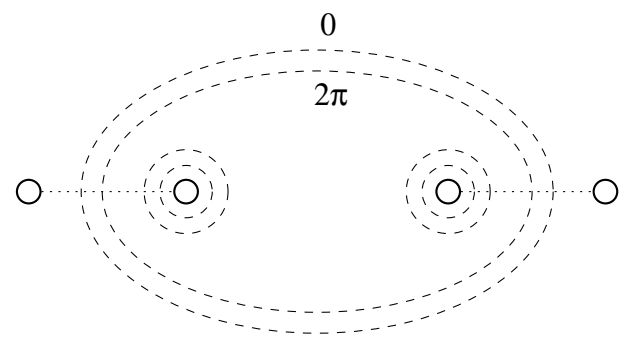

(b)

Fig. 2.5: The handle slide is interpreted geometrically as the full monodromy of two handles followed by a rotation of $2 \pi$ of each handle. The figure shows two equivalent representations for the handle slide: In (a), the geon is viewed as a rectangular box on the plane. In (b), we have identified two edges of the rectangle and the geon is represented as two circles on the plane.

where $\mathbf{c}_{1}=a_{1} b_{1} a_{1}^{-1} b_{1}^{-1}$. This operator is equivalent to braiding operators for particles and also satisfies the Yang-Baxter equation,

$$
(\mathcal{R} \otimes \mathbb{I})(\mathbb{I} \otimes \mathcal{R})(\mathcal{R} \otimes \mathbb{I})=(\mathbb{I} \otimes \mathcal{R})(\mathcal{R} \otimes \mathbb{I})(\mathbb{I} \otimes \mathcal{R}) .
$$

One can verify that the exchange operator (2.24) may be written as the product

$$
\mathcal{R}=\sigma R
$$

where $R \in \mathcal{A}^{(1)} \otimes \mathcal{A}^{(1)}$ is the analogue of the universal $R$-matrix for a quasi-triangular Hopf algebra. In our case $R$ is given by

$$
R=\sum_{a, b} Q_{(a, b)} \otimes \hat{\delta}_{a b a^{-1} b^{-1}}^{-1}
$$

The handle slide $\mathcal{S}$ is shown in Fig. 2.5. In (a), the geon is viewed as a rectangular box on the plane. In (b), we have identified two edges of the rectangle and the geon is represented as two circles on the plane connected by dotted lines. The action of $\mathcal{S}$ on the states and its presentation in terms of the other operators is given in [6].

This completes the description of $\mathcal{A}^{(2)}$. The algebra for two geons is generated by the elements of $\mathcal{A}^{(1)} \otimes \mathcal{A}^{(1)}, \mathcal{R}$ and the handle slide $\mathcal{S}$.

These constructions can be easily generalized to write down the algebra $\mathcal{A}^{(n)}$ for $n$ geons [6]. 


\section{Quantization}

The algebra $\mathcal{A}^{(1)}$ describes the topological degrees of freedom for a single geon on the plane. To quantize the system we need to find an irreducible representation of $\mathcal{A}^{(1)}$ on a Hilbert space $\mathcal{H}$. However, this Hilbert space will branch into irreducible representations of the field algebra:

$$
\mathcal{H}=\oplus_{r} \mathcal{H}_{r},
$$

where $\mathcal{H}_{r}$ denotes a particular irreducible representation describing a certain geon type. The algebra is finite dimensional, and therefore there will be a finite number of irreducible representations of $\mathcal{A}^{(1)}$. Furthermore, the Hilbert spaces $\mathcal{H}$ are all finite dimensional. Each representation gives us a possible one-geon sector of the theory.

In the case of quantum doubles, the irreducible representations are fully classified. See for instance ref. [22]. For the case of geons, the algebra is more complicated because

of the existence of internal structure. Nevertheless, the representations of $\mathcal{A}^{(1)}$ are quite similar to the ones of the quantum double of a finite group. This is not totally surprising, since in a certain limit, as discussed in the previous section, we recover the quantum double $\mathcal{D}^{(1)} \cong D(H)$. Actually, we can define a class of algebras $\mathcal{A}$, called transformation group algebras, that can have its representations classified and that are generic enough to contain the quantum double and the algebra $\mathcal{A}^{(1)}$ as particular cases. In the spirit of [22, one can then get all representations of $\mathcal{A}$.

Definition: Let $X$ be a finite set and $G$ a finite group acting on $X$. In other words, there is a map $\alpha_{g}: X \rightarrow X$ for each $g \in G$. As usual, we denote by $\mathcal{F}(X)$ the algebra of functions on $X$ and by $\mathbb{C}(G)$ the group algebra of $G$. We define the algebra $\mathcal{A}$ as the vector space

$$
\mathcal{A}:=\mathcal{F}(X) \otimes \mathbb{C}(G)
$$

with basis elements denoted by $\left(Q_{x}, g\right), Q_{x} \in \mathcal{F}(X)$ and $g \in \mathbb{C}(G)$, and the multiplication

$$
\left(Q_{x}, g\right) \cdot\left(Q_{y}, h\right):=\left(Q_{x} Q_{\alpha_{g}(y)}, g h\right) .
$$

Here, $Q_{x}$ is the characteristic function supported at $x \in X$. Let $x_{0}$ be an element of $X$. We denote by $K_{x_{0}} \subset G$ the stability subgroup with respect to $x_{0}$, i.e.,

$$
K_{x_{0}}=\left\{g \in G \mid \alpha_{g}\left(x_{0}\right)=x_{0}\right\}
$$

The stability subgroup $K_{x_{0}}$ divides the group $G$ into equivalence classes of left cosets. Let $N$ be the number of equivalence classes and let us choose a representative $\xi_{i} \in G$, $i=1 \ldots N$ for each class, with the convention that $\xi_{1}=e$. We can write the following partition of $G$ into left cosets:

$$
G=\xi_{1} K_{x_{0}} \cup \xi_{2} K_{x_{0}} \cup \ldots \cup \xi_{N} K_{x_{0}}
$$

We can now state the following result. 
Theorem Let $|j\rangle_{\rho}, j=1 \ldots n$ be a basis of a subspace $V_{\rho}$ of $\mathbb{C}(G)$ carrying an IRR $\rho$ of $K_{x_{0}}$. Then, for (a fixed) $x_{0} \in X$, elements $\xi_{i} \in G, i=1 \ldots N$ and $|j\rangle_{\rho} \in \mathbb{C}(G), j=1 \ldots n$ as stated above, the vectors

$$
\xi_{i}\left|x_{0}\right\rangle \otimes|j\rangle_{\rho}:=\left|\alpha_{\xi_{i}}\left(x_{0}\right)\right\rangle \otimes|j\rangle_{\rho}
$$

form a basis for an IRR of the algebra $\mathcal{A}$, given by

$$
\left(Q_{x}, g\right)\left|\alpha_{\xi_{i}}\left(x_{0}\right)\right\rangle \otimes|j\rangle_{\rho}:=\delta_{x, \alpha_{\xi_{i^{\prime}}}\left(x_{0}\right)}\left|\alpha_{\xi_{i^{\prime}}}\left(x_{0}\right)\right\rangle \otimes \Gamma^{(\rho)}(\beta)_{k j}|k\rangle_{\rho}
$$

where $\xi_{i^{\prime}}$ and $\beta$ are uniquely determined by the equation

$$
g \xi_{i}=\xi_{i^{\prime}} \beta
$$

and $\Gamma^{(\rho)}$ is the matrix for the representation $\rho$.

This result follows from a standard construction in induced representation theory (cf. discussion of the Poincaré group in [23]).

The quantum double $D(H)$ and the algebra $\mathcal{A}^{(1)}$ are particular cases of transformation group algebras. The quantum double is obtained by taking $X=H, G=H$, with the action $\alpha_{g}(h)=g h g^{-1}$. As for the algebra of a single geon, one takes

$$
X=H \times H
$$

and for the group $G$ the product $H \times \mathcal{M}$. The actions of $\hat{\delta}_{g} \in H$ and $W \in \mathcal{M}$ commute and are given by

$$
\alpha_{g}(a, b)=\left(g a g^{-1}, g b g^{-1}\right), \quad g \in H
$$

and

$$
\alpha_{W}(a, b)=\left(w^{(a)}, w^{(b)}\right), \quad W \in \mathcal{M},
$$

where we have used the notation of (2.18). The IRR's for the algebra (2.19) can be constructed given an element $(a, b) \in H \times H$. The stability subgroup $K_{(a, b)} \subset H \times H$ is defined by

$$
K_{(a, b)}=\left\{(g, W) \in H \times \mathcal{M} \mid \alpha_{g} \alpha_{W}(a, b):=\left(g w^{(a)} g^{-1}, g w^{(b)} g^{-1}\right)=(a, b)\right\} .
$$

Then, after choosing representatives $\xi_{1}, \ldots, \xi_{N}$ for the left cosets, the partition of $H \times \mathcal{M}$ can be written as

$$
H \times \mathcal{M}=\xi_{1} K_{(a, b)} \cup \xi_{2} K_{(a, b)} \cup \cdots \cup \xi_{N} K_{(a, b)} .
$$

Let $|1\rangle, \ldots,|n\rangle \in \mathbb{C}(H \times \mathcal{M})$ be a basis of an IRR of $K_{(a, b)}$. Then, according to the theorem, the vectors

$$
\left|\alpha_{\xi_{i}}(a, b)\right\rangle \otimes|j\rangle_{\rho}
$$

with $i=1 \ldots N, j=1 \ldots n$, form a basis of an IRR of the algebra $\mathcal{A}^{(1)}$. 
Let us express the representations of $\mathcal{A}^{(1)}$ in a more compact notation. The action of $H \times \mathcal{M}$ on $X=H \times H$ divides $X$ into orbits. We denote by $[a, b]$ the orbit containing the element $(a, b) \in H \times H$. We will collectivelly call $\rho$ the quantum numbers labeling the IRR's of $K_{(a, b)}$. One can see from (3.7) that an IRR $r$ is characterized by a pair $r=([a, b], \rho)$. A basis for an IRR $r$ of $\mathcal{A}^{(1)}$ will therefore be written as vectors $|i, j\rangle_{r}^{(a, b)}$ $, i=1, \ldots, N ; j=1, \ldots, n$ defined by

$$
|i, j\rangle_{r}^{(a, b)}:=\xi_{i}|a, b\rangle \otimes|j\rangle_{\rho}
$$

where $|a, b\rangle$ is a state in the defining representation, $\xi_{i}$ are the same as in (3.6) and $|j\rangle_{\rho}$ are base elements in the irreducible representations $\rho$ of $K_{(a, b)}$. Of course, the set of vectors thus defined depend on the pair $(a, b)$ we choose. We fix an $a$ and a $b$, and henceforth omit the superscript.

The action of $Q_{\left(a^{\prime}, b^{\prime}\right)}$ is given by

$$
Q_{\left(a^{\prime}, b^{\prime}\right)}\left|i, j>_{r}=Q_{\left(a^{\prime}, b^{\prime}\right)} \xi_{i}\right| a, b>\otimes\left|j>_{\rho}=Q_{\left(a^{\prime}, b^{\prime}\right)}\right| a_{i}, b_{i}>\otimes\left|j>_{\rho}=\delta_{a^{\prime}, a_{i}} \delta_{b^{\prime}, b_{i}}\right| i, j>_{r} .
$$

Let $\hat{\delta}_{g} W$ be a generic element of $H \times \mathcal{M}$. The equation

$$
\hat{\delta}_{g} W \xi_{i}=\xi_{i^{\prime}} \beta
$$

defines uniquely a new class $\xi_{i^{\prime}}$, together with an element of the stability group $\beta \in K_{(a, b)}$. The action of $\hat{\delta}_{g} W \in \mathcal{A}^{(1)}$ on $|i, j\rangle_{r}$ is determined by (3.10) and it reads

$$
\begin{aligned}
\hat{\delta}_{g} W|i, j\rangle_{r} & =\xi_{i^{\prime}}|a, b\rangle \otimes \beta|j\rangle_{\rho}= \\
& =\sum_{k} \Gamma^{(\rho)}(\beta)_{k j}\left|i^{\prime}, k\right\rangle_{r}
\end{aligned}
$$

where $\Gamma^{(\rho)}$ is the matrix representation of $K_{(a, b)}$.

Each IRR $r=([a, b], \rho)$ describes a distinct quantum geon. The corresponding vector spaces $\mathcal{H}_{r}$ generated by states $|i, j\rangle_{r}$, are all finite dimensional. Therefore we can easily make it into a Hilbert space by introducing the scalar product

$$
\left\langle i^{\prime}, j^{\prime} \mid i, j\right\rangle_{r}=\delta_{i i^{\prime}} \delta_{j j^{\prime}}
$$

Since the algebras $\mathcal{A}^{(1)}$ are not the same for different choices of the discrete group $H$, we cannot say in general what is the spectrum of a geon. First, we need to fix a group $H$ and then compute the spectrum for the corresponding $\mathcal{A}^{(1)}$.

Consider now two geons described by representations $r_{1}$ and $r_{2}$. The associated Hilbert space of states is simply

$$
\mathcal{H}^{(12)}:=\mathcal{H}_{r_{1}} \otimes \mathcal{H}_{r_{2}}
$$

As explained in Section 2 2 , the field algebra consists of $\mathcal{A}^{(1)} \otimes \mathcal{A}^{(1)}$ together with $\mathcal{R}$ and $\mathcal{S}$. The elements of $\mathcal{A}^{(1)} \otimes \mathcal{A}^{(1)}$ act naturally on (3.13). It remains to be said what is the action of $\mathcal{R}$ and $\mathcal{S}$ on states in $\mathcal{H}_{r_{1}} \otimes \mathcal{H}_{r_{2}}$. 
The action of $\mathcal{R}$ is completely determined by the formula (2.26):

$$
\mathcal{R}=\sigma \sum_{a, b} Q_{(a, b)} \otimes \hat{\delta}_{a b a^{-1} b^{-1}}^{-1} .
$$

In other words

$$
\mathcal{R}|i, j\rangle_{r_{1}} \otimes|k, l\rangle_{r_{2}}=\sum_{a, b} \hat{\delta}_{a b a^{-1} b^{-1}}^{-1}|k, l\rangle_{r_{2}} \otimes Q_{(a, b)}|i, j\rangle_{r_{1}}
$$

The generalization for $n$ geons is straightforward.

We may think of $\mathcal{R}$ and $\mathcal{S}$ as scattering matrices for a pair of geons. The $\mathcal{R}$-matrix represents an "elastic" interaction in the sense that two incoming geons of quantum numbers $r_{1}$ and $r_{2}$ are scattered into two objects carrying the same quantum numbers $r_{1}$ and $r_{2}$. The handle slide $\mathcal{S}$ on the contrary is a nontrivial scattering, each one of the two outgoing geons being a superposition of many geons in the spectrum.

\section{The Spin-Statistics Connection for $(2+1) d$ Topo- logical Geons}

The spin-statistics theorem is a well-established law of physics, and it holds true for most of the quantum particles in nature. In considering quantum topology change and, more generally, quantum gravity, one is naturally led to inquire whether a sort of field theory exists which gives geons as quantum excitations on a topologically trivial classical background. Such a theory remains hitherto utterly elusive, but one may try to investigate some of its aspects. For instance, in such a theory the quanta would be geons, so one fundamental research work would be a thorough analysis of the spin and statistics of geons and in particular whether the geons enjoy the canonical spin-statistics connection.

In order to pose the problem properly, we start with some general comments. Spin and statistics are two properties which can be defined independently of each other. They

refer not only to particles, but in general to localized sub-systems that may consist of several particles or even extended objects like solitons. The tensorial or spinorial nature of spin is determined by the behavior of the wave function when the sub-system undergoes a $2 \pi$ rotation. Statistics determines what happens when two identical sub-systems are exchanged. The canonical spin-statistics connection asserts the identity of the operators implementing $2 \pi$-rotation and exchange. It is thus clear that our first task is to define the quantum operators responsible for $2 \pi$ rotation and exchange. In the case of $(2+1) d$ orientable geons, we can easily find these operators among the algebra described in Section 2. In doing so, we will be led to a definite spin-statistics relation differing from the canonical one by a phase. 
In the usual spin-statistics theorem, we prepare a two-particle state which is a tensor product of two copies of the same (arbitrary) one-particle state with the same spin, in order to probe the relation between the exchange and $2 \pi$-rotation operators. However, here we meet a problem: we have only been able to prove the existence of such a relation in the special case when the total fluxes of each geon not only belong to the same conjugation class, but are the same. This is rather unexpected at first sight, because in the definition of the IRR's of the one-geon algebra, eq. (3.8), we can see that each vector of the basis of a representation space has an associated and possibly distinct flux $c_{i}=\xi_{i} c \xi_{i}^{-1}$, where $c=a b a^{-1} b^{-1}$. The geon state is in general a superposition of such vectors, and therefore the flux of a geon state is not well defined for most states in a given representation. To overcome this problem we advance the claim that the total flux should be a superselected quantity in the same sense that electric charge is superselected. We shall explain how this superselection occurs in Section 5. Here we simply note that as a consequence, the only physical states are superpositions of basis vectors with the same total flux. If we assume this, then the spin-statistics connection holds for all physical states.

We start by considering a pair of geons on the plane. The algebra $\mathcal{A}^{(2)}$ is fixed as soon as we choose a finite group $H$. In Section 2 we saw that the exchange of two geons is realized by the operator $\mathcal{R}$ defined in (2.26). Similarly, the $2 \pi$ rotation of one of the geons can be written as $\hat{C}_{2 \pi} \otimes \mathbb{I}$ or $\mathbb{I} \otimes \hat{C}_{2 \pi}$. We would like to know if the algebra $\mathcal{A}^{(2)}$ implies any kind of relation between exchange and $2 \pi$ rotations.

Let us start by fixing a representation $r=([a, b], \rho)$ of $\mathcal{A}^{(1)}$. State vectors $|i, j\rangle_{r} \in \mathcal{H}_{r}$ given by (3.8) do not have a well-defined spin, or in other words, they are not eigenstates of $\hat{C}_{2 \pi}$. In order to establish the spin-statistics relation we need first to know what are the eigenstates of $\hat{C}_{2 \pi}$. We recall that $\hat{C}_{2 \pi} \in \mathcal{M}$ and that $\mathcal{M}$ is a finite group. Therefore there exists a least non-negative integer $N$ such that

$$
\hat{C}_{2 \pi}^{N}=1
$$

and the eigenvalues of $\hat{C}_{2 \pi}$ are $e^{i 2 \pi s}$, where the spin $s=0,1 / N, 2 / N, \ldots,(N-1) / N$. One can see that the operator

$$
P_{s}=\frac{1}{N} \sum_{n=0}^{N-1} e^{-i n 2 \pi s} \hat{C}_{2 \pi}^{n}
$$

projects into states with spin $s$. The front factor is just a normalization constant to ensure that $P_{s}^{2}=P_{s}$, and we assume of course that $N \geq 2$, so that we have a non-trivial behavior under $2 \pi$-rotation. Furthermore, any state of $\mathcal{H}_{r}$ with spin $s$ can be obtained by linear combinations of $|s ; i j\rangle$ given by

$$
|s ; i, j\rangle=P_{s}|i j\rangle_{r}
$$

Consider a pair of geons both carrying the same representation $r=([a, b], \rho)$ and the same spin $s$. In addition, let us consider both geons to have the same total flux $c_{i}=\xi_{i} c \xi_{i}^{-1}$, where $c=a b a^{-1} b^{-1}$, i.e., we assume that each geon is in a state which is the superposition of basis vectors having the same flux. This will have the following consequence. The $\xi_{i}$ 's 
in (3.7) act on $|a, b\rangle$ in such a way that the flux $c$ becomes conjugated by $\xi_{i}$. In other words, the flux of each vector in the basis $\left\{|i, j\rangle_{r}\right\}$ will be equal to $c_{i}$. Hence $c_{i}$ becomes a quantity characterizing the particular superselection sector within $r$.We are going to show that the statistics of the system is completely determined by the spin $s$ and the representation $r$. It does not depend on $c_{i}$. More precisely,

$$
\mathcal{R}|\psi\rangle \otimes|\phi\rangle=e^{i\left(2 \pi s-\theta_{r}\right)}|\phi\rangle \otimes|\psi\rangle,
$$

where the states $|\psi\rangle$ and $|\phi\rangle$ transform according to the same represention $r$ and have both the same spin $s$ and the same total flux $c_{i}$. Also, $\theta_{r}$ is an angle that depends only on the representation $r$. The usual spin-statistics connection is true only for representations $r$ such that $\theta_{r}=0$.

It is enough to show that (4.4) is true for states $|\psi\rangle$ and $|\phi\rangle$ in the basis (4.3):

$$
\begin{aligned}
|\psi\rangle=P_{s}|i j\rangle_{r} & =\frac{1}{N} \sum_{n=0}^{N-1} e^{-i n 2 \pi s} \hat{C}_{2 \pi}^{n}\left\{\xi_{i}|a, b\rangle \otimes|j\rangle_{\rho}\right\} \\
|\phi\rangle=P_{s}|k l\rangle_{r} & =\frac{1}{N} \sum_{n=0}^{N-1} e^{-i n 2 \pi s} \hat{C}_{2 \pi}^{n}\left\{\xi_{k}|a, b\rangle \otimes|l\rangle_{\rho}\right\} \\
c_{i} & =c_{k} .
\end{aligned}
$$

Using (3.14) one can show after some algebra that

$$
\mathcal{R}\left\{P_{s}|i j\rangle_{r} \otimes P_{s}|k l\rangle_{r}\right\}=|\Phi\rangle \otimes P_{s}|i j\rangle_{r}
$$

with

$$
|\Phi\rangle=\frac{1}{N} \sum_{n} e^{-i n 2 \pi s} \hat{C}_{2 \pi}^{n} \hat{\delta}_{c_{i}}^{-1}\left\{\left|a_{k}, b_{k}\right\rangle \otimes|l\rangle_{\rho}\right\} .
$$

In the last formula, we used the notation

$$
\left|a_{k}, b_{k}\right\rangle:=\xi_{k}|a, b\rangle
$$

with $c_{i}$, the total flux of $\left|a_{i}, b_{i}\right\rangle$, being given by $c_{i}=a_{i} b_{i} a_{i}^{-1} b_{i}^{-1}$. The result (4.6) is a tensor product where only the first factor is not yet in the desirable form of (a phase) $\times|\psi\rangle$.

Let us take a closer look at $|\Phi\rangle$. First let us define

$$
E_{i}=\hat{\delta}_{c_{i}} \hat{C}_{2 \pi}
$$

and write $|\Phi\rangle$ as

$$
|\Phi\rangle=e^{i 2 \pi s} \frac{1}{N} \sum_{n} e^{-i n 2 \pi s} \hat{C}_{2 \pi}^{n} E_{i}^{-1}\left\{\xi_{k}|a, b\rangle \otimes|l\rangle_{\rho}\right\} .
$$

To proceed, we will show that

$$
E_{i} \xi_{k}=\xi_{k} E_{1}
$$


where $E_{1}=\hat{\delta}_{c} \hat{C}_{2 \pi}$ with $c=a b a^{-1} b^{-1}$. Indeed, first note that

$$
E_{i} \xi_{k}\left|a, b>=\hat{\delta}_{c_{i}} C_{2 \pi} \xi_{k}\right| a, b>=\hat{\delta}_{c_{i}} C_{2 \pi}\left|a_{k}, b_{k}>=\right| a_{k}, b_{k}>=\xi_{k} \mid a, b>,
$$

where we have used the fact that $c_{i}=c_{k}$. Therefore

$$
E_{i} \xi_{k}=\xi_{k} \beta
$$

where $\beta$ belongs to the stability group $K_{(a, b)}$ of $\mid a, b>$.

All we have to do is to show that in fact $\beta=E_{1}:=\hat{\delta}_{c} C_{2 \pi}$. We recall that by definition $\xi_{1}=e$.

From (4.13) it follows that

$$
\beta=\xi_{k}^{-1} E_{i} \xi_{k}
$$

If we take a generic $\xi_{k}$ to be of the form $\hat{\delta}_{g_{k}} \omega_{k}$ for some $g_{k} \in H$ and $\omega_{k} \in \mathcal{M}$, we see that

$$
\beta=\omega_{k}^{-1} \hat{\delta}_{g_{k}}^{-1}\left(\hat{\delta}_{c_{i}} C_{2 \pi}\right) \delta_{g_{k}} \omega_{k}=\hat{\delta}_{g_{k}}^{-1}\left(\hat{\delta}_{c_{i}} C_{2 \pi}\right) \hat{\delta}_{g_{k}}=\hat{\delta}_{g_{k}^{-1} c_{i} g_{k}} C_{2 \pi},
$$

since $\omega_{k}$ commutes with $H$-transformations. We will show presently that

$$
c_{i}=c_{k}=g_{k} c g_{k}^{-1}
$$

which allows us to conclude, from (4.15), that $\beta=E_{1}$, and hence (4.11) follows. In order to show (4.16) we write

$$
\left|a_{k}, b_{k}>=\xi_{k}\right| a, b>:=\hat{\delta}_{g_{k}} \omega_{k}\left|a, b>=\omega_{k}\right| g_{k} a g_{k}^{-1}, g_{k} b g_{k}^{-1}>.
$$

Remembering that the elements of $\mathcal{M}$ do not change the total flux, we can compare the total fluxes of the first and last expressions. That gives us

$$
c_{k}=g_{k} c g_{k}^{-1} \text {. }
$$

Now, from (4.11) we have

$$
E_{i}^{-1} \xi_{k}=\xi_{k} E_{1}^{-1}
$$

It is a simple exercise to show that $E_{1}$ is in the center of the stability group $K_{(a, b)}$ of $|a, b\rangle$. Therefore, from (3.11), we can write

$$
E_{i}^{-1}\left\{\xi_{k}|a, b\rangle \otimes|l\rangle_{\rho}\right\}=\xi_{k}|a, b\rangle \otimes E_{1}^{-1}|l\rangle_{\rho} .
$$

Since the states $|l\rangle_{\rho}$ form a basis for an (unitary) IRR of $K_{(a, b)}$, from Schur's lemma we conclude that the operator $E_{1}$ gives a phase $e^{i \theta_{r}}$ in (4.20) depending on the IRR $r=([a, b], \rho)$. Putting the results together we have

$$
|\Phi\rangle=e^{i\left(2 \pi s-\theta_{r}\right)} \frac{1}{N} \sum_{n} e^{-i n 2 \pi s} \hat{C}_{2 \pi}^{n}\left\{\xi_{k}|a, b\rangle \otimes|l\rangle_{\rho}\right\}=e^{i\left(2 \pi s-\theta_{r}\right)} P_{s}|k l\rangle .
$$

This concludes the demonstration of equation (4.4). Since the phase $\theta_{r}$ comes from general considerations, it is indeterminate in our formalism. 


\section{The Role of $H$ : Superselection and Clustering}

In this short Section we are interested in emphasizing how the hypothesis of superselection of total fluxes and the possibity of clustering of geon subsystems arise naturally if we confine ourselves to local operators as the only ones with physical significance.

We therefore start off by noting that the $H$-transformations in our one-geon algebra are actually non-local operations, since they are effectively equivalent, from the standpoint of the one geon, to move "distant geons", or alternatively, "distant fluxes" in a circle at infinity around the geon. This apparently innocent observation entails a striking conclusion: since all elements of the algebra other than the $H$-transformations (which elements are also "local operators"), commute with the total flux, we have that the total flux must be a superselected quantity. As we saw in the previous Section, this is enough to ensure the Spin-Statistics connection for geons. This may appear to be strange at first: we have seen that the total flux can be changed by the action of $H$. However, since $H$-transformations are non-local, they cannot affect local observables in a sensible, local theory. The only local operators would therefore be the elements of the mapping class group, and these clearly do not change the total flux. This can be generalized for $N$ geons: the total flux of the entire $N$-geon system is then superselected, the flux of each geon being however subject to changes, much as it occurs in $Q E D$ charged sectors. However, in this case one must have a suitable notion of clustering, i.e., if we consider $N-1$ geons to be "distant" in a suitable sense, the remaining "solitary" geon can be seen as really isolated, and it must be in a state belonging to a one-geon algebra IRR. We will explain the necessity of these concepts in what follows.

First of all we must make precise what our notion of "distant" is. We should recall that we are actually studying the low-energy limit of a field theory, and in spite of the fact that the theory in this limit is effectively independent of the metric, we have started off with a metric in order to define our theory. Therefore it makes sense to use this metric to measure distances and assume "distant" geons or fluxes to be those which are much further than the characteristic size of the geon under consideration. We hereafter take this to be the meaning of "distant". "Local" will then mean within distances comparable to the characteristic size of the geon.

With these definitions we can now proceed to clarify how the notions of clustering and superselection should arise to ensure the spin-statistics connection for geons. To establish this connection in the previous Section we considered states of the form $|\psi\rangle \otimes|\phi\rangle$, where $|\psi\rangle$ and $|\phi\rangle$ were states transforming in the same IRR $r$ and having the same spin $s$. We made, in the course of our demonstration, two basic asumptions:

- The states $|\psi\rangle$ and $|\phi\rangle$ were considered to have definite, well-defined total fluxes $c_{i}$ and $c_{k}$, i.e., they were each superpositions of basis vectors having the same total flux;

- The fluxes $c_{i}$ and $c_{k}$ are the same. 
The second asumption is very natural and easily implemented, since as pointed out in 24] in a similar context, the fluxes "being equal" is a well-defined, $H$-invariant notion. One only has to prepare two one-geon states with same total flux. The first asumption, however, looks quite artificial at first sight, without some extra input. After all, each IRR vector space is generated by vectors having different total fluxes, although in the same conjugacy class. To impose that only superpositions of vectors having the same flux should be considered apparently threatens generality. This is not so however, if we assume that the notions of clustering of geon subsystems and superselection of total flux should play a role in our physical description. First, let us consider the clustering property. Consider a system of $N$ geons on the plane. It is really rather natural to assume that geon subsystems can be isolated as long as we consider only local operators. This means that, if we fix one geon and take the other $N-1$ geons to infinity, this remaining geon should be described by the one-geon algebra $\mathcal{A}^{(1)}$, and its state should belong to an IRR of $\mathcal{A}^{(1)}$. On the other hand, the total flux of the $N$-geon system is superselected, but the total fluxes of individual geons are not uniquely defined by the $N$-geon total flux, and in particular a geon can even be in a superposition of various flux states. It is to preclude this possibility that the clustering property appears: since the geon can be isolated, it is effectively equivalent to a one-geon system, and in particular all operations which change its flux are indistinguishable from $H$-transformations on a one-geon system. Therefore the isolated geon in a pure state must have a definite total flux as well. (Impure one-geon states with a probability distribution of total fluxes are of course permitted).

The consequence of the previous discussion for a two-geon system is as follows. For a one-geon system, superselection of the total flux means that the IRR's $r$ must further split into superselected subspaces, and the physical states belong to these subspaces. Now, for a two-geon system in a state $|\psi\rangle \otimes|\phi\rangle$, only the total flux must be superselected, but the clustering property ensures that each geon must have a definite flux. Therefore for

physical states, $|\psi\rangle$ and $|\phi\rangle$ must separately be superpositions of states with definite flux. The conclusion is that the two asumptions we made to check the spin-statistics connections become most natural if we include superselection of the total flux and the clustering property as physical requirements of the theory.

\section{Geons in Quantum Gravity}

In this Section our aim is to describe what are the consequences of our results to quantum gravity. In the canonical metric formalism of gravity on a spacetime manifold $\mathcal{M}$ of the form $\Sigma \times \mathbb{R}$, we may perform a space versus time splitting and define the classical configuration space $Q$ as follows. Let us specialize to $(2+1) d$. Let $R^{\infty}(\Sigma)$ be the space of all Riemannian metrics on the space manifold $\Sigma$ which are equal to some conical metric 
in a neighborhood $\mathcal{N}$ of infinity円. We quotient this space by the group $\operatorname{Diff} f^{\infty}(\Sigma)$ of diffeomorphisms of the spatial 2-manifold $\Sigma$ to obtain $Q$ [21]. Therefore we may denote the $Q$ as

$$
Q=\frac{R^{\infty}(\Sigma)}{\operatorname{Diff} f^{\infty}(\Sigma)}
$$

Now, $Q$ is not simply-connected in general, and in this case one may have many distinct quantizations, a phenomenon which is seen for example in the " $\theta$ vacua" of QCD?. To see this we resort to the so-called covering space quantization, which we briefly review here (for a more complete account, see Ref. [21]). In this approach, wave functions (defining domains of operators like the Hamiltonian) are taken to be functions on the universal covering space $\tilde{Q}$ of $Q$ with certain specific properties. It can be shown that in our case

$$
\tilde{Q}=\frac{R^{\infty}(\Sigma)}{\operatorname{Diff} f_{0}^{\infty}(\Sigma)}
$$

where $\operatorname{Dif} f_{0}^{\infty}(\Sigma)$ is the component connected to the identity, and hence a normal subgroup, of $\operatorname{Dif} f^{\infty}(\Sigma)$. In what follows it will always be understood that these diffeos are of $\Sigma$, and hence we will omit the argument.

It is well known that the universal covering $\tilde{X}$ of any topological space $X$ is a principal bundle over $X$ with structure group $\pi_{1}(X)$, and the projection $p: \tilde{X} \rightarrow X$ given by the covering map. The structure group has a free right action $\gamma: \tilde{x} \rightarrow \tilde{x} \gamma$ on $\tilde{X}$ and the quotient $\tilde{X} / \pi_{1}(X)$ by this action is again $X$. This action is fiber-preserving, i.e., for every $\gamma \in \pi_{1}(X), \tilde{x} \in \tilde{X}$ we have $p(\tilde{x} \gamma)=p(\tilde{x})$. Therefore $\tilde{Q}$ is a principal bundle over $Q$ with structure group $\pi_{1}(Q)$, which can inferred from (6.1) and (6.2) to be isomorphic to the mapping class group $M_{\Sigma}$.

Wave functions $\Psi$ need not be single-valued on $Q$ if $Q$ contains non-contractible loops. Actually the transformation of the wave function when such loops are traversed gives a representation of $\pi_{1}(Q)$. This can be seen in the following way. All that must be singlevalued are observables like $\Psi^{*} \Psi$. On the other hand, since the universal covering space is by definition simply-connected, functions on it can always be taken to be single-valued. Therefore, if we could define wave functions as functions on $\tilde{Q}$ such that $\Psi^{*} \Psi$ is still a function on $Q$, we would circumvent the multi-valuedness of the wave functions while leaving the probability interpretation unharmed. This task is easily accomplished in the following way. Let $\mathcal{H}_{\rho}$ be a complex vector space with a hermitian inner product $\langle$, carrying a unitary representation $\rho$ of $\pi_{1}(Q) \simeq M_{\Sigma}$. We say that a function $\Psi: \tilde{Q} \rightarrow \mathcal{H}_{\rho}$ is equivariant if for every $\gamma \in \pi_{1}(Q), \tilde{q} \in \tilde{Q}$ we have

$$
\Psi(\tilde{q} \gamma)=\rho\left(\gamma^{-1}\right) \Psi(\tilde{q})
$$

\footnotetext{
${ }^{1}$ These boundary conditions for the metric substitute the usual $(3+1) d$ "asymptotically Minkowskian" scenario for the $(2+1) d$ case. See [25] and references therein for a more complete discussion.

${ }^{2}$ In this section, the word "quantization" will have a meaning slightly different from the rest of the paper. It will mean simply an appropriate assignment of a Hilbert space of wave functions to a classical configuration space.
} 
Hence, since $\tilde{Q} / \pi_{1}(Q)=Q$, we have that $\langle\Psi, \Psi\rangle$ can indeed be viewed as a function on $Q$, as we wanted. Therefore our quantum Hilbert space $V_{\rho}$ may be taken to be (the norm completion of) the space of equivariant functions $\Psi: \tilde{Q} \rightarrow \mathcal{H}_{\rho}$ such that the function $\langle\Psi, \Psi\rangle$, seen as a function on $Q$, gives a finite number when properly integrated on the whole of $Q$ (the latter process defines the inner product). This recipe obviously depends on which representation $\rho$ we take. Actually, it can be shown [21] that there are at least as many inequivalent quantizations as there are unitary irreducible representations of $M_{\Sigma}$.

As we have mentioned, wave functions on $Q$ pick an "Aharonov-Bohm phase" whenever they traverse a non-contractible path in $Q$, i.e., they transform according to some representation of $\pi_{1}(Q)$. Since this group is non-abelian in general, the wave function may transform via a non-abelian representation. This is akin to the behaviour of sections of a vector bundle with a flat connection. Actually this is the case: it is well-known (see e.g., [26]) that the set of equivariant functions on a principal bundle taking values in some vector space $V$ is in bijective correspondence to the set of sections of the associated vector bundle with fiber $V$. Since the structure group is discrete, the bundle is always flat.

We therefore arrive at the conclusion that any unitary representation of the mapping class group provides a vector bundle over $Q$ whose space of square-integrable sections forms a possible Hilbert space for geons in quantum gravity as far as kinematics is concerned. These spaces must have further imposed dynamical constraints, i.e., they must be a suitable arena for dynamics before they really can be claimed to be authentic quantum gravity Hilbert spaces for geons. In this $(2+1) d$ context we can actually do more than this. It is possible to impose all the dynamical constraints of general relativity to obtain the reduced configuration space, thereby taking into account dynamical aspects as well. Our space $Q$ will then be the moduli space of the surface $\Sigma$, and its universal covering $\tilde{Q}$ will be the Teichmuller space [27]. Again, $\pi_{1}(Q)$ will be the mapping class group and our discussion will go mostly along the same lines [ This state of affairs is somewhat reminiscent of quantization of matter particles in Minkowski spacetime, in the context of usual quantum mechanics. One can have many representations of the Lorentz group, but on the one hand only some of them seem to be realized in nature, and on the other hand the dynamics selects which representation survives in each physical situation, e.g., the tensorial representations for the Klein-Gordon field or the spinorial and tensorial representations for the Dirac field.

Thus, we are led to the questions of whether, and how, the Hilbert spaces we have presented in the previous Sections fit into this scheme, and in particular whether the spinstatistics connection we have found extends to these quantum gravity geon states. First of all, we note that some of the Hilbert spaces $\mathcal{H}_{r}$, carrying representations $r=([a, b], \rho)$ of our field algebra $\mathcal{A}$ (in the notation defined in Section 5) carry naturally a representation of the mapping class group: we recall that the elements of this group were naturally included in $\mathcal{A}$, and hence we may say that $r$ contains a representation (possibly reducible) of the mapping class group. We will again denote this representation by $r$. Therefore the vector

\footnotetext{
${ }^{3}$ Strictly speaking, this discussion is valid for genus $g \geq 2$. For genus 1 , things are a bit more complicated. For details see, for instance, 28.
} 
bundle associated to $\tilde{Q}$ with fiber $\mathcal{H}_{r}$, where the $r$ 's are just as stated, gives another Hilbert space for geons in quantum gravity, via its square-integrable sections. In this new scenario the fibers are internal state spaces.

However, we are now faced with another problem: do these sections carry a representation of the mapping class group? In other words, is it possible to extend the action on each fiber to an action on the space of sections? The answer is known to be negative in general [19, 29]. One can nevertheless implement operators corresponding to elements of $M_{\Sigma}$ on states localized at a point $q \in Q$. In particular, we may extend the operators $\mathcal{R}$ and $C_{2 \pi}$, related to statistics and spin respectively, to operators $\hat{R}$ and $\hat{C}_{2 \pi}$ acting on such localized states. We will show at the end of this Section that such states obey a spin-statistics connection inherited from the fibers. Here we presently give a simple geometrical discussion to bring out the reasoning behind these remarks.

A very useful construction of the universal covering space $\tilde{Q}$ is as follows [21]. Let us assume that the space $Q$ is connected (if this is not so we can always choose a connected component). Let $q_{0}$ be a point of $Q$ which once chosen is not to be changed. Let $\alpha_{q}$ be a path on $Q$ from $q_{0}$ to another point $q \in Q$ t. The path space $\mathcal{P} Q$ of $Q$ is the space $\left\{\alpha_{q}\right\}$ of these paths. Let us next say that two paths $\alpha_{q}$ and $\alpha_{q}^{\prime}$ are equivalent and write $\alpha_{q} \sim \alpha_{q}^{\prime}$ if one of them can be deformed to the other holding $q_{0}$ and $q$ fixed. One can then show that the space $\tilde{Q}$ is the same as the space of equivalence classes $\left[\alpha_{q}\right]$ of such paths, the projection map $p: \tilde{Q} \rightarrow Q$ being given by $\left[\alpha_{q}\right] \mapsto q$. For a fixed $q$, these equivalence classes form the fiber over the point $q$.

The group $\pi_{1}(Q)$ can be identified with the set of equivalence classes of loops starting and ending at $q_{0}$, i.e., $\left[\alpha_{q_{0}}\right]$, with the compositions of loops $\left[\alpha_{q_{0}}^{\prime}\right]\left[\alpha_{q_{0}}\right]=\left[\alpha_{q_{0}}^{\prime} \circ \alpha_{q_{0}}\right]$, where we first trace $\left[\alpha_{q_{0}}\right]$, and then trace $\left[\alpha_{q_{0}}^{\prime}\right]$. This group acts on $\tilde{Q}$ on the right via the composition of paths: $\left[\alpha_{q}\right]\left[\alpha_{q_{0}}\right]=\left[\alpha_{q} \circ \alpha_{q_{0}}\right]$. This corresponds to the free, fiberwise action of $\pi_{1}(Q)$ on $\tilde{Q}$ that we mentioned before. Note moreover that we have chosen a fiducial point and put $\pi_{1}(Q) \simeq \pi_{1}\left(Q ; q_{0}\right)$, the latter denoting the homotopy group of paths based at $q_{0}$, to be our "model" for the structure group. Now, we might consider $\pi_{1}(Q ; q)$, the homotopy group of paths based at another point $q \in Q$. This group is isomorphic to $\pi_{1}\left(Q ; q_{0}\right)$, but the isomorphism is not canonical, and we will see that this fact prevents the extension of the action of $\pi_{1}(Q)$ when this group is non-abelian. We point out that $\pi_{1}(Q ; q)$ acts on the space $\left\{\left[\alpha_{q}\right]\right\}$ on the left, and therefore does not interfere with the action of $\pi_{1}\left(Q ; q_{0}\right)$; if we denote an element of $\pi_{1}(Q ; q)$ by $\left[\gamma_{q}\right]$, we have that $\left[\gamma_{q}\right]\left[\alpha_{q}\right]=\left[\gamma_{q} \circ \alpha_{q}\right]$.

We now show that exchange and $2 \pi$-rotation correspond to actions like that of $\pi_{1}(Q ; q)$, and not to the globally defined right action of $\pi_{1}(Q)$. If $d$ denotes a diffeo and $h$ a metric, let $h d$ denote the pull-back metric $d^{*} h$. Then $\tilde{Q}$ consists of elements $h$ Diff $f_{0}^{\infty}, Q$ of elements $h$ Diff $f^{\infty}$, and $\pi_{1}(Q)=\operatorname{Diff} f^{\infty} / \operatorname{Dif} f_{0}^{\infty}$ acts on $\tilde{Q}$ on the right : $h$ Dif $f_{0}^{\infty} \rightarrow$

\footnotetext{
${ }^{4}$ In this discussion all parametrized curves $\left\{\alpha:[0,1] \rightarrow Q \mid \alpha(0)=q_{0} ; \alpha(1)=q\right\}$ with different parametrizations but with same image in $Q$ are to be regarded as the same path.

${ }^{5}$ Let $G$ be any group and $X$ a space on which $G$ acts on the right. We may take the quotient $X / G$ of equivalence classes by the action. Let also $x \in X$. We denote by $x G$ the equivalence class of $x$ in $X / G$.
} 
$\left(h \operatorname{Dif} f_{0}^{\infty}\right)\left(\operatorname{dDif} f_{0}^{\infty}\right)=h d \operatorname{Dif} f_{0}^{\infty}$, with $d \in \operatorname{Diff} f^{\infty}$. This action is globally defined. The association of a $2 \pi$-rotation or exchange however is not to this action of $\operatorname{Diff} f^{\infty} / \operatorname{Dif} f_{0}^{\infty}$. Instead, it is obtained as follows. Let $q=h D i f f^{\infty} \in Q$ correspond to two well-separated geons and let $\{q(t): 0 \leq t \leq 1\}$ be a loop in $Q$ based at $q(0)=q(1)=q$. We can write $q(t)=h(t) D i f f^{\infty}$, and there exists an element $d \in D i f f^{\infty}$ such that $h(1)=h(0) d$, since $q(0)=q(1)$. Now it is shown elsewhere [19] that the physical process of exchange say is associated with an element $\mathcal{R} D$ if $f_{0}^{\infty}$ of Diff ${ }^{\infty} / \operatorname{Diff}_{0}^{\infty}\left(\mathcal{R} \in\right.$ Diff $\left.f^{\infty}\right)$ and a loop based at $q(0) \in Q$. But this correspondence is not unique, being deduced from the $\pi_{1}(Q ; q)$ action on $\tilde{Q}$. Thus according to [19], the exchange process gives a curve $\{h(t)\}$ with $h(1)=h(0) \mathcal{R}$. It becomes the curve $\left\{h(t) D i f f_{0}^{\infty}\right\}$ in $\tilde{Q}$ and the loop $\left\{h(t) D i f f^{\infty}\right\}$ in $Q$. Now to find the diffeo and the loop for exchange based at $q(0)$, the starting metric can be $h(0)$ or $\bar{h}(0)=h(0) d$, for any $d \in D i f f^{\infty}$ as both give rise to the same $q(0)$. Then for the two curves $\{h(t)\}$ and $\{\bar{h}(t)\}$, we have $h(1)=h(0) \mathcal{R}$ and $\bar{h}(1)=\bar{h}(0) \mathcal{R}$. They give the curves $\left\{h(t) D i f f_{0}^{\infty}\right\}$ and $\left\{\bar{h}(t) D i f f_{0}^{\infty}\right\}$ in $\tilde{Q}$ and the loops $\left\{h(t) D i f f^{\infty}\right\}$ and $\left\{\bar{h}(t) D i f f^{\infty}\right\}$ in $Q$. But the homotopy classes of these loops are not in general the same, being related by the action of $\pi_{1}(Q ; q)$. They do not therefore always define an unambiguous element of $\tilde{Q}$. To see this first note that $\left\{\bar{h}(t) d^{-1} D i f f_{0}^{\infty}\right\}$ also projects to the loop $\left\{\bar{h}(t) D i f f^{\infty}\right\}$ while at the same time it has the same starting point $h(0) \operatorname{Diff} f_{0}^{\infty}$ as $\left\{h(t) D i f f_{0}^{\infty}\right\}$. It follows that the lifts of the loops $\left\{h(t) D i f f^{\infty}\right\}$ and $\left\{\bar{h}(t) D i f f^{\infty}\right\}$ to $\tilde{Q}$ with the same starting point $h(0) D i f f_{0}^{\infty}$ are $\left\{h(t) D i f f_{0}^{\infty}\right\}$ and $\left\{\bar{h}(t) d^{-1} D_{\text {Dif }} f_{0}^{\infty}\right\}$. But their endpoints in general are different, being $h(0) \mathcal{R} D$ if $f_{0}^{\infty}$ and $h(0) d \mathcal{R} d^{-1} D$ if $f_{0}^{\infty}$ respectively, showing that the two loops may not be homotopic. Further the diffeos associated to the exchange can be $\mathcal{R}$ or $d \mathcal{R} d^{-1}$ and their images in Diff $f^{\infty} / \operatorname{Diff}_{0}^{\infty}$ can be different.

Suppose then that we want to consider a global action of $\pi_{1}(Q)$ on the quantum states. The two-geon configuration $h D$ if $f^{\infty} \equiv q \in Q$ is described in quantum theory by an equivariant function $\Psi$ on $\tilde{Q}$ taking values in some vector space $\mathcal{H}_{\Gamma}$ which carries a representation $\Gamma$ of $\operatorname{Diff} f^{\infty} / \operatorname{Diff} f_{0}^{\infty}$. We assume that $\Psi$ is localized at a point $\left[\alpha_{q}\right] \in \tilde{Q}$ in the fiber over $q$. The exchange process will then correspond to a loop class $\left[\gamma_{q}\right] \in \pi_{1}(Q ; q)$. This will act on the wave function by

$$
\left(\left[\gamma_{q}\right] \Psi\right)\left(\left[\alpha_{q}\right]\right)=\Psi\left(\left[\gamma_{q}\right]^{-1}\left[\alpha_{q}\right]\right) .
$$

Since $\left[\gamma_{q}\right]^{-1}\left[\alpha_{q}\right]=\left[\gamma_{q}^{-1} \circ \alpha_{q}\right]$ is in the fiber over $q$ as well, there exists a unique $\left[\sigma_{0}^{\gamma}\right] \in \pi_{1}(Q)$ such that $\left[\gamma_{q}^{-1} \circ \alpha_{q}\right]=\left[\alpha_{q}\right]\left[\sigma_{0}^{\gamma}\right]$. Therefore

$$
\Psi\left(\left[\gamma_{q}\right]^{-1}\left[\alpha_{q}\right]\right)=\Gamma\left(\left[\sigma_{0}^{\gamma}\right]^{-1}\right) \Psi\left(\left[\alpha_{q}\right]\right) .
$$

However, this association of an element of the fundamental group of $Q$ based at $q$ to an element of $\pi_{1}(Q)$ is canonical only for $\Psi$ localized at a point $\tilde{q}$ in the fiber over $q$ as stated, otherwise we may pick another $\left[\alpha_{q}^{\prime}\right] \in p^{-1}(q)$ which is related to $\left[\alpha_{q}\right]$ by the relation $\left[\alpha_{q}^{\prime}\right]=\left[\alpha_{q}\right][t]$, for some $[t] \in \pi_{1}(Q)$. Then we have

$$
\begin{aligned}
\left(\left[\gamma_{q}\right] \Psi\right)\left(\left[\alpha_{q}^{\prime}\right]\right)=\Psi\left(\left[\gamma_{q}\right]^{-1}\left[\alpha_{q}^{\prime}\right]\right) & =\Psi\left(\left[\gamma_{q}\right]^{-1}\left[\alpha_{q}\right][t]\right) \\
& =\Psi\left(\left[\alpha_{q}\right]\left[\sigma_{0}^{\gamma}\right][t]\right)=\Gamma\left([t]^{-1}\left[\sigma_{0}^{\gamma}\right]^{-1}[t]\right) \Psi\left(\left[\alpha_{q}^{\prime}\right]\right) .
\end{aligned}
$$


Thus we have problems with continuity of the action for non-abelian $\Gamma$ if we try to extend the action of $\pi_{1}(Q)$ to non-localized states.

We are now ready to consider the content of the spin-statistics theorem in this context of localized states. Let $\mathcal{H}_{\Gamma}$ be a Hilbert space carrying a representation $\Gamma$ of the algebra for one geon. Consider the space of equivariant functions $\Psi: \tilde{Q} \rightarrow \mathcal{H}_{\Gamma}$, which is the space of states of the geon in quantum gravity, and denote it by $V_{\Gamma}$. We have seen that the mapping class group will not act on all of $V_{\Gamma}$, but will have an action on localized states. Technically speaking these are "delta functions" concentrated at some point $\tilde{q} \in \tilde{Q}$. This means distributions, i.e., linear functionals $\delta_{\tilde{q}}$ on the $\Psi$ 's such that, for each $\Psi \in V_{\Gamma}$, we have

$$
\delta_{\tilde{q}}(\Psi) \equiv \Psi(\tilde{q}) .
$$

Let $d \in \operatorname{Diff} f^{\infty} / \operatorname{Dif} f_{0}^{\infty}$, and $\tilde{q}=h \operatorname{Dif} f_{0}^{\infty}$, where as before $h$ denotes some metric on $\Sigma$. Then Diff $f^{\infty} /$ Dif $f_{0}^{\infty}$ acts on the localized space as follows:

$$
\hat{d} \delta_{\tilde{q}}=\delta_{\tilde{q} d^{-1}},
$$

and given any state $\Psi \in V_{\Gamma}, \delta_{\tilde{q} d^{-1}}(\Psi)=\Psi\left(\tilde{q} d^{-1}\right)=\Gamma(d) \delta_{\tilde{q}}(\Psi)$, from the equivariance property. Hence we may simply put, with a slight abuse of notation,

$$
\hat{d} \delta_{\tilde{q}}=\Gamma(d) \delta_{\tilde{q}}
$$

We are actually interested in the action of $\mathcal{R}$ and $C_{2 \pi}$. Like in the case of vectors in $\mathcal{H}_{\Gamma}$, we can construct localized states with definite spin $s$, namely by applying the spin projector $P_{s}$ in (4.2) to any generic state. Note, nevertheless that the definition given in (6.8) does not apply directly in this case, since $P_{s}$ is not an element of the mapping class group as it has been defined. This needs not bother us, though, since the definition of (6.9) extends by linearity to the algebra generated by the mapping class group, so we may define a localized state of spin $s$, denoted by $\delta_{\tilde{q}}^{(s)}$ through the equation

$$
\delta_{\tilde{q}}^{(s)}=\Gamma\left(P_{s}\right) \delta_{\tilde{q}}
$$

Now pick two equal states $\delta_{\tilde{q}}^{(s)}$ localized at the same point $\tilde{q} \in \tilde{Q}$ and with the same spin $s$. These are eigenvectors of the operator $\hat{C_{2 \pi}}$ with eigenvalue $\exp i 2 \pi s$, and we may write, for any two states $\Phi$ and $\Psi$ in $V_{\Gamma}$ :

$$
\hat{\mathcal{R}} \delta_{\tilde{q}}^{(s)} \otimes \delta_{\tilde{q}}^{(s)}(\Phi, \Psi)=\mathcal{R}\left\{\Gamma\left(P_{s}\right) \Phi(\tilde{q}) \otimes \Gamma\left(P_{s}\right) \Psi(\tilde{q})\right\} .
$$

But since the vectors in the parenthesis are both vectors in $\mathcal{H}_{\Gamma}$, the spin-statistics relation is valid for them. Since $\Phi$ and $\Psi$ are arbitrary, we may conclude from (4.4) that

$$
\hat{\mathcal{R}} \delta_{\tilde{q}}^{(s)} \otimes \delta_{\tilde{q}}^{(s)}=e^{i\left(2 \pi s-\theta_{r}\right)} \delta_{\tilde{q}}^{(s)} \otimes \delta_{\tilde{q}}^{(s)},
$$

which is the expression for the spin-statistics connection for the localized geon states in quantum gravity. 


\section{$7 \quad$ Final Remarks}

In this paper, we have shown how to explicitly encode information on non-trivial spatial topology in the quantum theory of topological geons. We have described its classical degrees of freedom by using the low-energy limit of a Yang-Mills theory coupled to a Higgs field in the Higgs phase, where the symmetry is spontaneously broken down to a finite gauge group. It has been argued that this is enough to capture aspects of the topology of the underlying space manifold, and it has been shown how such a theory can be quantized. The field algebra for one or many geons has been derived, and its representations, corresponding to the various geonic sectors of the theory, have been worked out in detail.

Our discussion borrowed heavily from the theory of quantization of vortices [13. It led to a striking consequence: we have been able to derive a new spin-statistics relation obeyed by the geonic states. In this relation, there is still a parameter $\theta_{r}$ to be fixed for

each representation of the geon algebra $\mathcal{A}^{(1)}$, but to obtain it explicitly one has to to fix a finite group $H$ and use the formalism to work out the representations of the geon algebra. We will attempt this elsewhere. We have also shown how the geonic states we describe here correspond to quantum gravity states of geons.

One is naturally led to inquire whether the framework developed in this paper can be extended to cover the more general case when the gauge group is a Lie group. In particular it is known that general relativity in $(2+1) d$ can be viewed as a Chern-Simons theory with gauge group $I S O(2,1)$ [30], and therefore one may investigate the spin-statistics connection when $H$ in this paper is replaced by $I S O(2,1)$. Such generalizations will be the issues of a forthcoming paper.

\section{Acknowledgments}

We would like to thank J.C.A. Barata, B.G.C. da Cunha, A. Momen and S. Vaidya for many helpful discussions. The work of A.P. Balachandran was supported by the Department of Energy, U.S.A., under contract number DE-FG02-85ERR40231. The work of E. Batista, I.P. Costa e Silva and P. Teotonio-Sobrinho was supported by FAPESP, CAPES and CNPq respectively. A.P.B. acknowledges the wonderful hospitality of Denjoe O'Connor and support of CINVESTAV at Mexico, D.F. while this work was being completed. 


\section{References}

[1] R. Sorkin: "Introduction to Topological Geons", in Topological Properties and Global Structure of Spacetime, edited by P.G. Bergmann and V. de Sabhata, Plenum, New York (1986).

[2] A.P. Balachandran, A. Daughton, Z.C. Gu, G. Marmo, R.D. Sorkin and A.M. Srivastava: Int. J. Mod. Phys. A8 (1993) 2993.

[3] J.L. Friedman and R.D. Sorkin: Phys. Rev. Lett. 44 (1980) 1100;

J.L. Friedman and R.D. Sorkin: Gen. Rel. Grav. 14 (1982) 615.

[4] H.F. Dowker and R.D. Sorkin: "A Spin-Statistics Theorem for Certain Topological Geons",gr-qc/9609064.

[5] R.D. Sorkin and S. Surya: "Geon Statistics and UIRs of the Mapping Class Group", hep-th/9704081.

[6] A.P. Balachandran, E. Batista, I.P. Costa e Silva and P. Teotonio-Sobrinho: "Quantum Topology Change in $(2+1) d "$, hep-th/9905136.

[7] A.P. Balachandran, G. Bimonte, G. Marmo, A. Simoni: Nucl. Phys. B446 (1995) 299-314, gr-qc/9503046.

[8] D. Marolf: Phys.Lett. B392(1997)287-290, gr-qc/960203.

[9] N. Seiberg and E. Witten: Nucl. Phys. B426 (1994) 19.

[10] A.P. Balachandran and P. Teotonio-Sobrinho: Int. Jour. Mod. Phys. A8(1993) 723752, hep-th/9205116.

[11] R. Haag: "Local Quantum Physics", Springer-Verlag (1992).

[12] T.H. Koornwinder and N.M. Muller: J. Lie Theory 7 (1997), 33-52;

T.H. Koornwinder, F.A. Bais and N.M. Muller: UvA-WINS-Wisk. 97-14 preprint, q-alg/9712042.

[13] M.D.F. de Wild Propitius and F.A. Bais: Discrete Gauge Theories Proc. CRMCAP Summer School "Particles and Fields 94", Banff, Springer Verlag (1996), hepth/9511201

M.D.F. de Wild Propitius: Topological Interactions in Broken Gauge Theories, PhD. thesis University of Amsterdam, 1995, hep-th/9511195.

[14] A.P. Balachandran, A. Momen, P. Teotonio-Sobrinho and S. Vaidya: "Topological Quantization and Quantum Groups", to appear.

[15] C. Kassel: "Quantum Groups", Springer-Verlag (1995). 
[16] M.V. Berry and J.M. Robbins: Proc. Roy. Soc. Lond. A 453 (1997) 1771.

[17] J. Anandan: "Relativistic Spin-Statistics Connection and Kaluza-Klein Space-Time", hep-th/9806070.

[18] I. Duck and E.C.G. Sudarshan: Am. J. Phys. 66 (1998) 284 and references therein; M.J. York: "Spatial Asymmetry for Particle Pairs and the Spin-Statistics Theorem", and references therein.

[19] C. Aneziris, A.P. Balachandran, M. Bourdeau. S. Jo, T.R. Ramadas and R.D. Sorkin: Mod. Phys. Lett. A4 (1989) 331;

C. Aneziris, A.P. Balachandran, M. Boureau, S. Jo, R.D. Sorkin and T.R. Ramadas: Int. Jour. Mod. Phys. A4 (1989) 5459.

[20] J.S. Birman: "Braids, Links and Mapping Class Groups", Princeton University Press (1972).

[21] A.P. Balachandran,, G. Marmo, B.S. Skagerstam and A. Stern: "Classical Topology and Quantum States", World Scientific (1991).

[22] K. Szlachányi and P. Vecsernyés: "Quantum Symmetry and Braid Group Statistics in G-Spin Models", Comm. Math. Phys. 156 (1993) 127.

[23] A.P. Balachandran and C.G. Trahern: "Lectures on Group Theory for Physicists", Monographs and Textbooks in Physical Science 3, Bibliopolis, Napoli (1985).

[24] H.-K. Lo and J. Preskill: Phys. Rev. D48 (1993) 4821.

[25] J. Samuel: Phys. Rev. Lett. 71 (1993) 215.

[26] D. Husemoller: "Fibre Bundles", 2nd ed., Springer Verlag, Berlin (1975).

[27] S. Carlip: "Lectures on (2+1) Dimensional Gravity", gr-qc/9503024.

[28] J. Louko and D.M. Marolf: Class. Quantum Grav. 11 (1994) 311.

[29] A.P Balachandran, G. Marmo, N. Mukunda, J.S. Nilsson, E.C.G. Sudarshan and F. Zaccara: Phys. Rev. Lett. 50 (1983) 1553; Phys. Rev. D29 (1984) 2919;

P. Nelson and A. Manohar: Phys. Rev. Lett. 50 (1983) 943.

[30] A. Achúcarro and P.K. Townsend, Phys. Lett. B180 (1986) 89;

E. Witten, Nucl. Phys. B311 (1988) 46. 